\title{
Pontormo und Pontano
}

\section{Zu Paolo Giovios Programm für die beiden Lünettenfresken in Poggio a Caiano}

\author{
Christina Strunck
}

Im großen und ganzen läßt sich das gesamte Bildprogramm des zentralen Festsaales in der MediciVilla Poggio a Caiano unter einem einzigen Begriff subsumieren, mit einem einzigen Wort umschreiben. Das Schlüsselwort lautet Glovis. „Glovis“? Nach Auskunft Giovios erwählte Giuliano de’ Medici diese zusammenhanglose Buchstabenfolge, die er auf einem antiken epigraphischen Fragment in Rom erblickt hatte, zu seiner glückverheißenden Imprese (wohl nicht ahnend, daß er seine Fortüne so mit einer antiken Kloakeninschrift verknüpfte): $\mathrm{Da}$ nämlich „Glovis“ rückwärts gelesen „si $\operatorname{volg}(\mathrm{e})$ “ („es wendet sich“) heißt, sah Giuliano darin einen Hinweis auf das Rad der Fortuna bzw. auf das Glück, das sich ihm wieder zuwende. ${ }^{1}$

Im Salone von Poggio a Caiano nimmt das Schriftfeld „Glovis“, rund wie ein Glücksrad, keinen großen, aber einen zentralen Platz unter einem der beiden Okulusfenster ein (Abb. 1). In diesem magischen Wort bündeln sich quasi all jene zyklischen Prozesse, die die Ausmalung des Saales ausführlich schildern: Die Wandfresken beschwören den großen historischen Kreislauf, indem sich in antiken Geschichtsbildern Anspielungen auf die Rolle der Medici als Erben der klassischen virtus verstecken, während in Pontormos Lünettenbild die Zyklen der Natur dazu dienen, die Regeneration der schwer angeschlagenen Medici-Dynastie zu veranschaulichen, deren Schicksal sich in der bildlichen Fiktion wieder zum Guten wendet. ${ }^{2}$

„Glovis“ läßt sich jedoch auch auf die Geschichte jenes Saales selbst beziehen, die reich an Fortunawechseln war. Die erste, 1519 begonnene Ausstattungsphase fand ein abruptes Ende, als der Auftraggeber, Medici-Papst Leo X., 1521 verstarb. Nachdem ein Projekt zur Komplettierung der Ausmalung (1532-1534/37) gescheitert war, er- folgte die Fertigstellung der Dekoration zu guter Letzt 1578-1582 durch Alessandro Allori.

Der über sechzigjährige Entstehungsprozeß erschwert das Verständnis des ohnehin komplizierten Bildprogrammes. Kliemann hat darauf hingewiesen, wie problematisch die Annahme ist, daß die von Alloris Berater Vincenzo Borghini formulierten Bildthemen dem ursprünglichen Projekt entsprechen, das Paolo Giovio ausarbeitete. ${ }^{3}$ Was aber entwarf Giovio für die später von Allori gestalteten Flächen?

Der vorliegende Beitrag zur Lösung dieses Rätsels konzentriert sich auf die beiden Lünetten. Da davon ausgegangen werden kann, daß die Themen der zwei einander gegenüberliegenden Lünettenfresken aufeinander bezogen waren, muß zuerst eine Betrachtung der von Pontormo 1521 fertiggestellten Südlünette (Abb. 1) erfolgen. Auf der Grundlage neuer Restaurierungsbefunde lassen sich der ikonographischen Ausdeutung jenes Werks einige Aspekte hinzufügen. Unter Rückgriff auf Vasaris Überlieferung und zwei Zeichnungen Pontormos soll dann versucht werden, das nördliche Lünettenbild zu rekonstruieren, als dessen literarische Quelle ich ein Gedicht von Giovanni Pontano vorstellen möchte.

Den concetto der Südlünette haben Winner und Kliemann mithilfe des Gedichtes ,Ne la Stagion entschlüsselt, das Ariost 1519 anläßlich der schweren Krankheit des jungen Lorenzo de' Medici schrieb. ${ }^{4}$ Darin klagt das wohl als Florentia zu verstehende lyrische Ich um einen kranken Lorbeerbaum. Das Wortspiel Lorbeer - lauro - Laurentius - Lorenzo war bereits in der Medici-Panegyrik unter Lorenzo il Magnifico üblich und wurde für dessen Enkel Lorenzo wieder aufgegriffen; ${ }^{5}$ natür- 


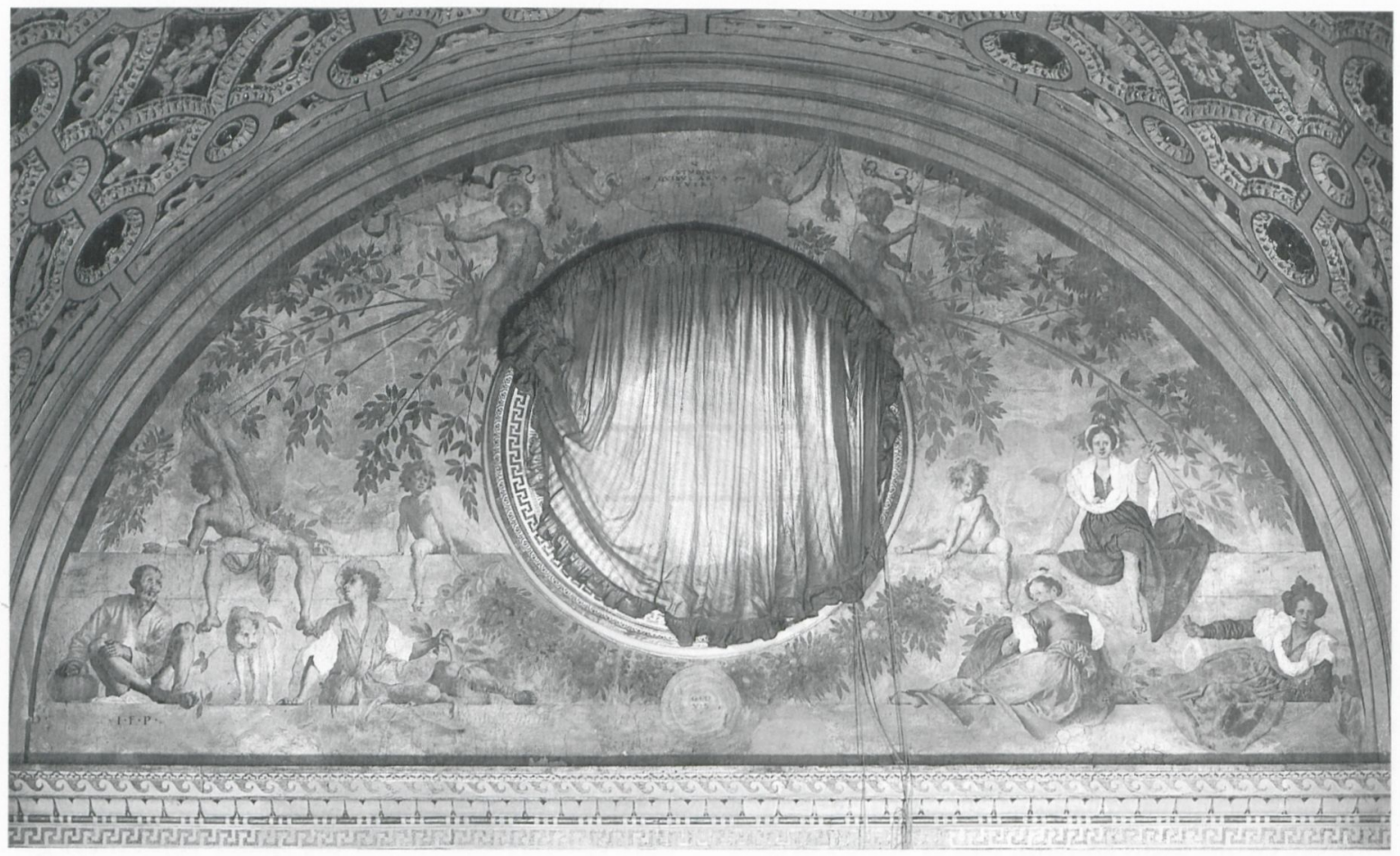

1 Pontormo, Lünettenfresko in der Sala grande (Südseite), Villa Medici, Poggio a Caiano

lich meint auch Ariost mit dem Lorbeerbaum den kranken Lorenzo. Dieser Lorbeerbaum wurde vom Winter so gebeutelt, daß nur noch ein Zweig Laub trägt. Nun fleht Florentia die Götter an, sie möchten dem Baum beistehen und verhindern, daß er ganz abstirbt, denn wenn der Lorbeer sterbe, sterbe auch sie selbst. ${ }^{6}$

Pontormos Fresko (Abb. 1) bildet quasi die Fortsetzung des Gedichtes: Putten sitzen auf zwei dicken abgesägten Baumstümpfen, aus denen junge Lorbeerzweige austreiben. Der Wunsch, den Ariost formulierte, hat sich hier bereits erfüllt: Die alten Äste sind zwar abgestorben, aber der Baum hat sich mit Hilfe der Götter erholt. Sie haben den Lorbeer beschnitten, damit er danach um so kräftiger austreibe: Tugend erstarkt durch Unglück, konnte man dies auslegen. ${ }^{7}$ Eine hohe Mauer hinterfing ursprünglich den kostbaren Lorbeer, um ihn vor künftigen Unbilden zu schützen. ${ }^{8}$ Obwohl Lorenzo, auf den sich Ariosts "Lauro" bezog, mittlerweile tot und seine Nachfolge überaus unsicher war, wollte das Fresko eine optimistische Botschaft vermitteln: Die Medici-Dynastie werde wie der Lorbeerbaum weiterleben.
Die Formulierung in Ariosts erwähntem Gedicht „soccorran tutti i dei, tutte le dee, / che de li arbori han cura, l'arbor mio" paraphrasiert einen Vers aus der Einleitung von Vergils, Georgica' (I, 21): „diique deaeque omnes, studium quibus arva tueri". ' Die zweite Hälfte dieses lateinischen Verses, der die Schutzgottheiten der Landwirtschaft anruft, erscheint in dem Schriftfeld über dem Okulusfenster der Lünette. Pontormos Fresko zeigt also jene Götter, die dem kranken Lorbeer zu Hilfe kamen. Aber welche?

Die Benennung der einzelnen Bildfiguren gestaltet sich schwierig, da eindeutige Attribute fehlen. Wichtige Deutungshinweise liefert Vasari in seinem Bericht über Pontormos Arbeit in Poggio a Caiano: „Avendo a fare un Vertunno con i suoi agricultori, fece un villano che siede con un pennato in mano, tanto bello e ben fatto, che è cosa rarissima, come anco sono certi putti che vi sono, oltre ogni credenza vivi e naturali. Dall'altra banda, facendo Pomona e Diana con altre dee, le aviluppò di panni forse troppo pienamente, nondimeno tutta l'opera è bella e molto lodata. " ${ }^{10}$

Pomona ist Schutzgöttin der Bäume, daher zur 
Pflege des Lorbeers ganz recht am Platz. ${ }^{11}$ Der wandelbare Vertumnus, der Pomona in den unterschiedlichsten Gestalten zu erobern versuchte, kann als Sinnbild für den Jahreslauf verstanden werden. Die verschiedenen Erscheinungsbilder des Vertumnus werden in den Ovid-Kommentaren als die einzelnen Jahreszeiten interpretiert; einer anderen Deutung zufolge steht Vertumnus für den Himmel und Pomona für die Erde, deren mehr oder weniger fruchtbares Verhältnis sich je nach Jahreszeit verändert. ${ }^{12}$ Zusammen symbolisieren die beiden Gottheiten jene zyklischen Prozesse in der Natur, die dem fast abgestorbenen $\mathrm{Me}-$ dici-Lorbeer zu hoffnungsvollen, neuen Trieben verholfen haben.

Die lange Zeit einhellig von der Forschung akzeptierte Meinung, bei dem Liebespaar Vertumnus und Pomona handle es sich um die Frau mit der kleinen Säge und den Greis, die symmetrisch an den äußersten Rändern der Lünette plaziert sind (Abb. 2 und 3), ${ }^{13}$ wurde in den letzten Jahren wiederholt angezweifelt. Falciani deutete 1992 den apollinischen Nackten auf der oberen Mauer als Vertumnus mit der Begründung, genau in dieser seiner eigentlichen Gestalt habe Vertumnus schließlich Pomonas Herz gewonnen: „Er erschien ihr so, wie die helleuchtende Sonne sich zeigt, wenn sie durch die Wolkendecke bricht und unverhüllt wieder erstrahlt. “14 Bei der jüngsten Restaurierung des Freskos kam zudem unterhalb des alten Mannes in der linken Bildecke, der bislang als „Vertumnus“ galt, der Schriftzug „PAN“ zum Vorschein. ${ }^{15}$ Der Hund neben dieser Figur bestätigt die Benennung, da er ein traditionelles Attribut des Pan ist. ${ }^{16}$ In der Antike wurde bei den Lupercalien, dem jährlichen Fest zu Ehren Pans, ein Hund geopfert, um Schutz vor Krankheiten sowie Fruchtbarkeit der Frauen, Felder und Herden zu erlangen. ${ }^{17}$ Das fügt sich perfekt dem Thema der Lünette ein, die ja das Fortleben einer von Krankheit und frühem Tod bedrohten Dynastie beschwört.

Vasaris Formulierung „Vertunno con i suoi agricoltori“ drückt aus, daß Vertumnus den anderen Männern übergeordnet ist. Dies entspricht der Position des Jünglings auf der Mauer (Abb. 2), der die Spitze einer Figurenpyramide bildet und der in seiner nackten Schönheit klar die auffälligste männliche Figur des Freskos ist. Auf ihn trifft Vasaris Charakterisierung „tanto bello“ sicherlich besser zu als auf den Alten am linken Bildrand.

Das kompositionelle Pendant des Jünglings, die Frau rechts vom Okulusfenster (Abb. 3), ist durch ihre exponierte Position und ihr leuchtend rotes Gewand als die weibliche Hauptfigur und Partnerin des Vertumnus gekennzeichnet. Wegen ihres Kranzes aus Ähren, Mohnblüten und Mohnkapseln wurde sie verschiedentlich als Ceres interpretiert. Diese Attribute bilden aber auch angemessene Kennzeichen Pomonas, denn in dem von Boccaccio imaginierten Garten der Pomona wachsen u. a. verschiedene Getreidesorten und Mohnblumen. ${ }^{18}$ Die Wahl jener Attribute veranschaulicht, daß „Ceres“ und „Pomona“ nur verschiedene $\mathrm{Na}$ men für dieselbe Sache, nämlich die personifizierte Erde, sind, wie Cartari postulierte. ${ }^{19}$

Gemeinsam verkörpern die beiden Hauptpersonen auf der oberen Mauer, Vertumnus und Pomona, deutlicher als das restliche Bildpersonal den concetto der Lünette: Sie drücken ihre Verbundenheit mit dem Lorbeerbaum aus, indem sie ihm räumlich besonders nahe sind bzw. seine Zweige $\mathrm{zu}$ sich heranziehen, und geben sich durch ihre breitbeinig-obszönen Posen als die Fruchtbarkeitsgötter zu erkennen, die den Bestand der Dynastie garantieren. ${ }^{20}$ Die vier Landleute in der unteren Bildhälfte spielen, wie Kliemann gezeigt hat, durch subtile Farbsymbolik auf die vier Jahreszeiten an: ${ }^{21}$ Nach einem gängigen Schema von rechts nach links angeordnet, ${ }^{22}$ sind Frühling und Sommer weiblich, Herbst und Winter männlich, was dem grammatischen Geschlecht von primavera, estate, autunno und inverno entspricht. Pan kommt passenderweise die Rolle des Winters zu, da sein Fest im Februar gefeiert wurde. ${ }^{23}$ Die von Vasari erwähnte Diana wäre eventuell analog mit dem Frühling in Verbindung zu bringen. ${ }^{24}$

Die ungewöhnliche Pose des Vertumnus erfordert eine Erklärung. Wie Vasari berichtet, zermarterte Pontormo sich wegen des Freskos für Poggio a Caiano das Gehirn und fertigte unzählige, immer wieder andere Vorzeichnungen an. ${ }^{25}$ Einige dieser Studien zeigen einen Jüngling, der sich mit der Hand die Augen beschirmt. ${ }^{26}$ Der Jüngling auf der Zeichnung Uffizien $6599 \mathrm{~F} \mathrm{r}$ (Abb. 4) gleicht in Haltung und Geste deutlich einem Hirten in Bal- 


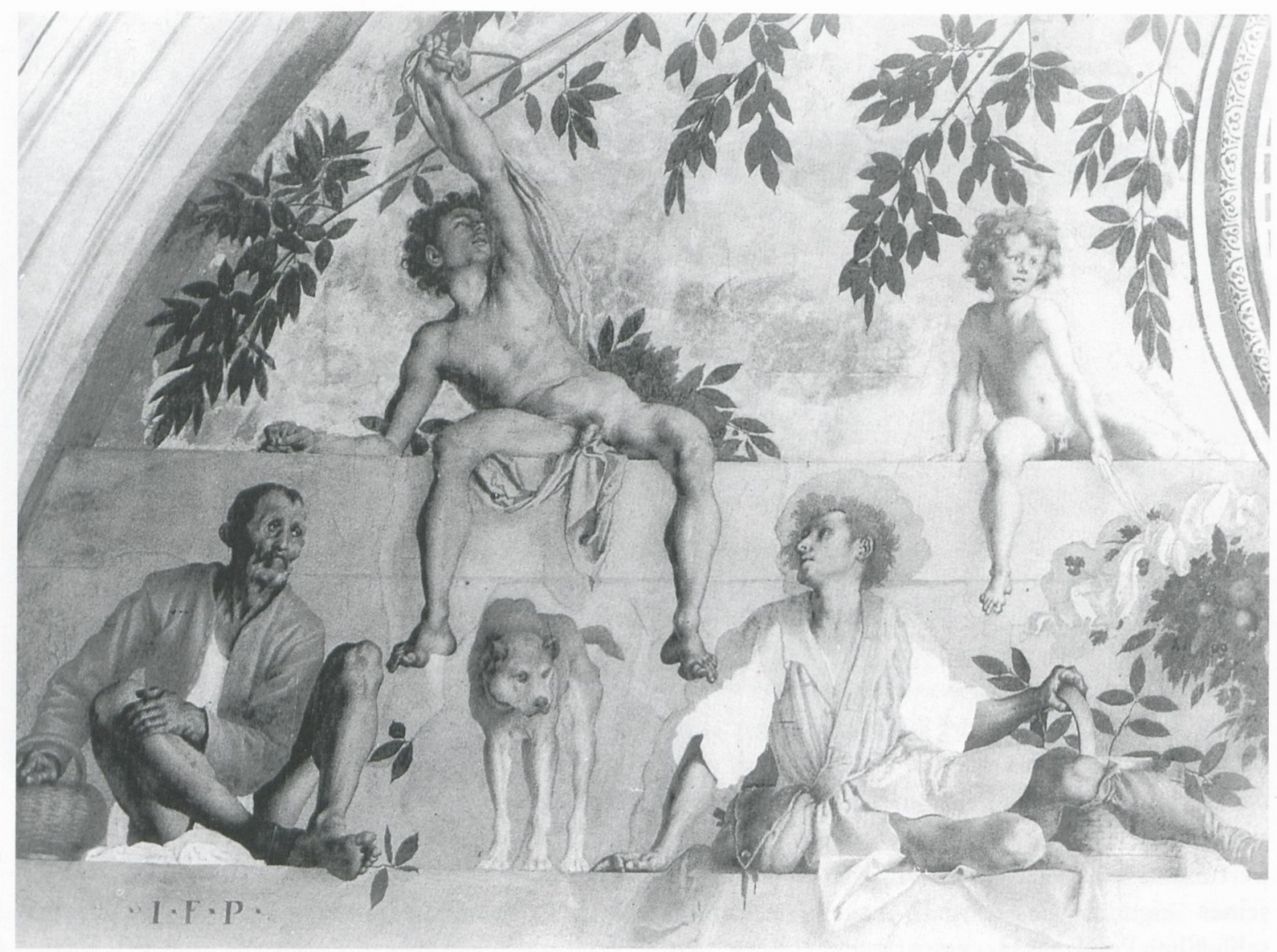

2 Pontormo, Lünettenfresko in der Sala grande (Südseite), Villa Medici, Poggio a Caiano, Detail

dovinettis ,Geburt Jesu'-Fresko (Abb. 5), das Pontormo bestens kannte. ${ }^{27}$ Bei Baldovinetti schützt der Hirte mit der an die Stirn gelegten Hand seine Augen vor der göttlichen Erscheinung des Verkündigungsengels. Schon in der antiken Kunst war das Verschatten der Augen (aposkopein) ein bekannter Epiphaniegestus. ${ }^{28}$ Pontormos Vertumnus variiert dieses in den Vorzeichnungen angelegte Motiv: Wie die Restaurierung eindeutig geklärt hat, packt Vertumnus mit seiner linken Hand nämlich keinen Zweig des Lorbeers, das heißt seine eigenartige Pose ist nicht von der Baumpflege bedingt; vielmehr beschattet er seine Augen mit Arm und Tuch (Abb. 2). ${ }^{29}$ Vielleicht ist es in diesem Kontext kein Zufall, daß seine Körperhaltung recht genau Michelangelos ,Jonas' kopiert, eine weitere Epiphaniedarstellung. ${ }^{30}$ Einen möglichen Grund für die motivischen Anleihen verrät die folgende formale Betrachtung.
Pontormo richtete die Lichtführung in dem Lünettenfresko auf die natürliche Lichtquelle aus, so daß die Figuren rechts des Okulusfensters von links, die Figuren links des Fensters dagegen von rechts beleuchtet erscheinen. Bei strenger Befolgung dieser Regel müßten jedoch die Putten über dem Okulus von unten und nur sehr schwach beleuchtet sein. Um dies zu vermeiden, führte Pontormo eine zweite, fiktive Lichtquelle ein, die über dem Scheitel der Lünette liegt. ${ }^{31}$ Dieses irreale Licht bezeichnet gleichzeitig auch eine überreale, göttliche Präsenz, wie die Reaktion des Vertumnus verdeutlicht: Er schaut mit staunend geöffnetem Mund und verzücktem Blick zu der Quelle des mysteriösen Lichts empor, weicht mit dem Oberkörper betroffen zurück, beschattet seine Augen nach dem Muster von Epiphaniedarstellungen. Neben den Landgöttern wirkt auch der Himmel selbst an der Wiederbelebung der Medici-Dynastie 




3 Pontormo, Lünettenfresko in der Sala grande (Südseite), Villa Medici, Poggio a Caiano, Detail

mit, erkennt er. Eine ähnliche Idee kam zum Beispiel beim Medici-Karneval von 1513 zum Ausdruck, wo die durch das Wiederausschlagen des Lorbeers symbolisierte Rückkehr der Familie aus dem Exil dem Eingreifen Gottes zugeschrieben wurde. ${ }^{32}$

Die neu entdeckte Inschrift „PAN“ fügt der Interpretation des Lünettenfreskos weitere Facetten hinzu. Pan ist aus Vergils Eklogen als der „deus Arcadiae" bekannt. ${ }^{33}$ In einem Kommentar zur zweiten Ekloge führte Servius aus, Pan symbolisiere den Kosmos, Erde und Himmel und den Kreislauf des Jahres; ähnliche Äußerungen finden sich bei Macrobius und in den Orphischen Hymnen. ${ }^{34}$ Auch in den seit den 1480er Jahren viel gelesenen Eklogen Sannazaros besitzt Pan die Doppelbedeutung als Sinnbild für den Kosmos und Herrscher Arkadiens. ${ }^{35}$ Beides spielte in der MediciPanegyrik eine Rolle. Bereits Cosimo il Vecchio war (über das schmeichelhafte Wortspiel Cosimo Cosmo) mit Pan, dem Kosmos, identifiziert worden. ${ }^{36}$ Marsilio Ficino übertrug diese Idee auf Lorenzo il Magnifico, dessen Villa bei Fiesole Poliziano als das arkadische Reich des Pan besang. ${ }^{37}$ Später schlüpfte Lorenzo d. J. in die Rolle Pans: Severus Minervius huldigte ihm 1516 als „pan Medica de gente satus “ ${ }^{38}$ Vor allem aber zur Zeit des Magnifico, der die Villa von Poggio a Caiano erbaute und dessen Sohn Leo X. das Freskenprogramm „per memoria di Lorenzo suo padre“ bestellte, ${ }^{39}$ begegnet Pan oft: in den Gedichten von Lorenzo und seinem Kreis, in philosophischen Diskursen sowie in Signorellis wohl für Lorenzo bestimmtem Pan-Gemälde. ${ }^{40}$

Der Pan des Lünettenfreskos in Poggio a Caiano konnte vor diesem Hintergrund vielfältige Assoziationen hervorrufen: Im Zusammenhang mit dem Wiederaustreiben des Lorbeers erschien Pan 


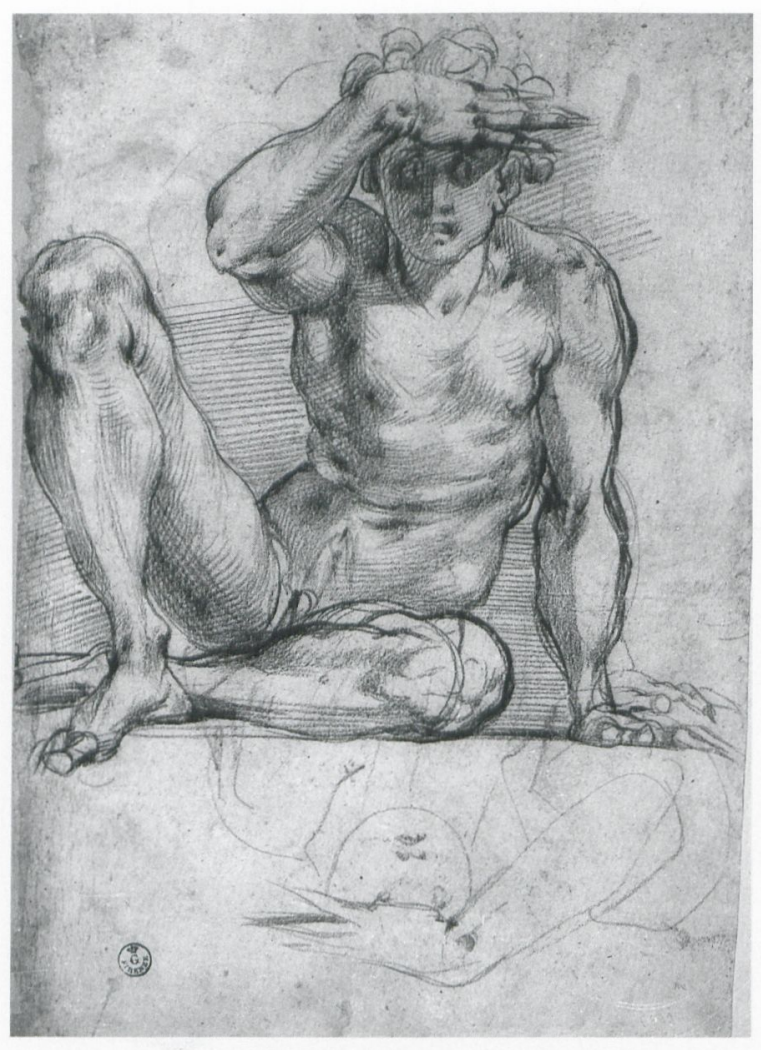

4 Pontormo, Vorstudie für den „Vertumnus“, Florenz, Uffizien, $6599 \mathrm{~F} \mathrm{r}$

als Gott des Jahreslaufes und als der Fruchtbarkeitsgott der Lupercalien, an die sein Attribut, der Hund, erinnerte. Wer mit dem Medici-Kreis gut vertraut war, konnte in Pan eine Hommage an den kosmisch-arkadischen Magnifico sehen, zu dessen Ehren der Saal ausgemalt wurde, dachte vielleicht sogar an Ficinos „Pan Saturnius“, den Schutzgott der philosophisch-kontemplativen villeggiatura. ${ }^{41}$ Die primäre, geläufigste Bedeutung Pans dürfte aber doch die des arkadischen Herrschers gewesen sein; seine Präsenz definiert den Schauplatz des Lünettenfreskos als Arkadien.

Arkadien - daran knüpft sich in der Renaissance die Idee eines idyllischen Landlebens, das nostalgisch die Lebensformen des Goldenen Zeitalters nachvollzieht. Arkadien und Goldenes Zeitalter lassen sich jedoch nicht gleichsetzen; die Arkadien-Dichtung formuliert stets die Wehmut über das Ende des Goldenen Zeitalters und die Hoffnung auf dessen Rückkehr. ${ }^{42}$

Im Goldenen Zeitalter produzierte die Erde noch alles Nötige von selbst, ohne daß sich jemand darum kümmern mußte, berichtet Lorenzo il Magnifico in Anlehnung an Ovid. ${ }^{43}$ Daher erzählt bei Boccaccio Pomona, daß sie zwar im Goldenen Zeitalter geboren, ihre Hilfe bei der Pflanzenpflege aber erst in späteren Jahrhunderten gebraucht wurde. ${ }^{44}$ Wenn in Pontormos Fresko der Lorbeer nicht von allein gedeiht, sondern unter Pomonas Aufsicht beschnitten werden muß, um wieder auszutreiben, spricht dies ebenso wie Pans Präsenz dafür, daß Pontormo keinen "golden age garden ", ${ }^{45}$ sondern ein mediceisches Arkadien darstellt. In der Medici-Propaganda wird jedoch oft das Neu-Ausschlagen des Lorbeers mit der Rückkehr des Goldenen Zeitalters verknüpft. ${ }^{46}$ Die arkadische Sehnsucht nach dem Goldenen Zeitalter erfüllt sich also demnächst, impliziert das Bild - sie sollte sich in der gegenüberliegenden Lünette erfüllen, worauf ich noch zurückkommen werde.

Die Hoffnung auf eine Wendung zum Besseren verdichtet sich in der eingangs erwähnten "Glovis"-Imprese, die sich auf den Kreislauf der Natur bzw. auf die mit ebensolcher Zwangsläufigkeit wieder zur Blüte gelangende Medici-Dynastie beziehen läßt. ${ }^{47}$ Die Vertumnus-Lünette kann so auch als Paolo Giovios Versuch begriffen werden, für "Glovis“ (eine Imprese, die nur aus Text besteht und damit gegen die von Giovio formulierten Regeln der Impresenkunst verstößt $)^{48}$ eine geeignete Impresen-pictura zu finden. Nun gehört es laut Giovio zu den Kennzeichen der Imprese, daß sie nur für Eingeweihte und nicht für jedermann („ogni plebeo“) zu entziffern sein soll. ${ }^{49}$ Genauso verhält es sich mit der Lünette: Die Götter erscheinen als Landleute verkleidet, die erklärende Beischrift „Pan“ wurde übermalt, damit der Sinn nicht zu offensichtlich auf der Hand lag. ${ }^{50}$

Im Gegensatz zu dieser bewußten Verrätselung posaunt Alloris gegenüberliegende Lünette (Abb. 6) ihre Botschaft überdeutlich heraus: Herkules im Garten der Hesperiden war ein altbekanntes, leicht verständliches Gleichnis für den tugendsamen Herrscher und sein blühendes Reich. ${ }^{51}$ Kann das Thema dieses Freskos, wie Cox-Rearick glaubt, ${ }^{52}$ also wirklich schon von Giovio gewählt worden sein? Schwarzenberg und Paolozzi Strozzi haben in ihrer Analyse von Alloris Werk dargelegt, daß die Rolle der Fortuna als „divina provvidenza“ darin charakteristisch für das spätere 16. Jahrhun- 


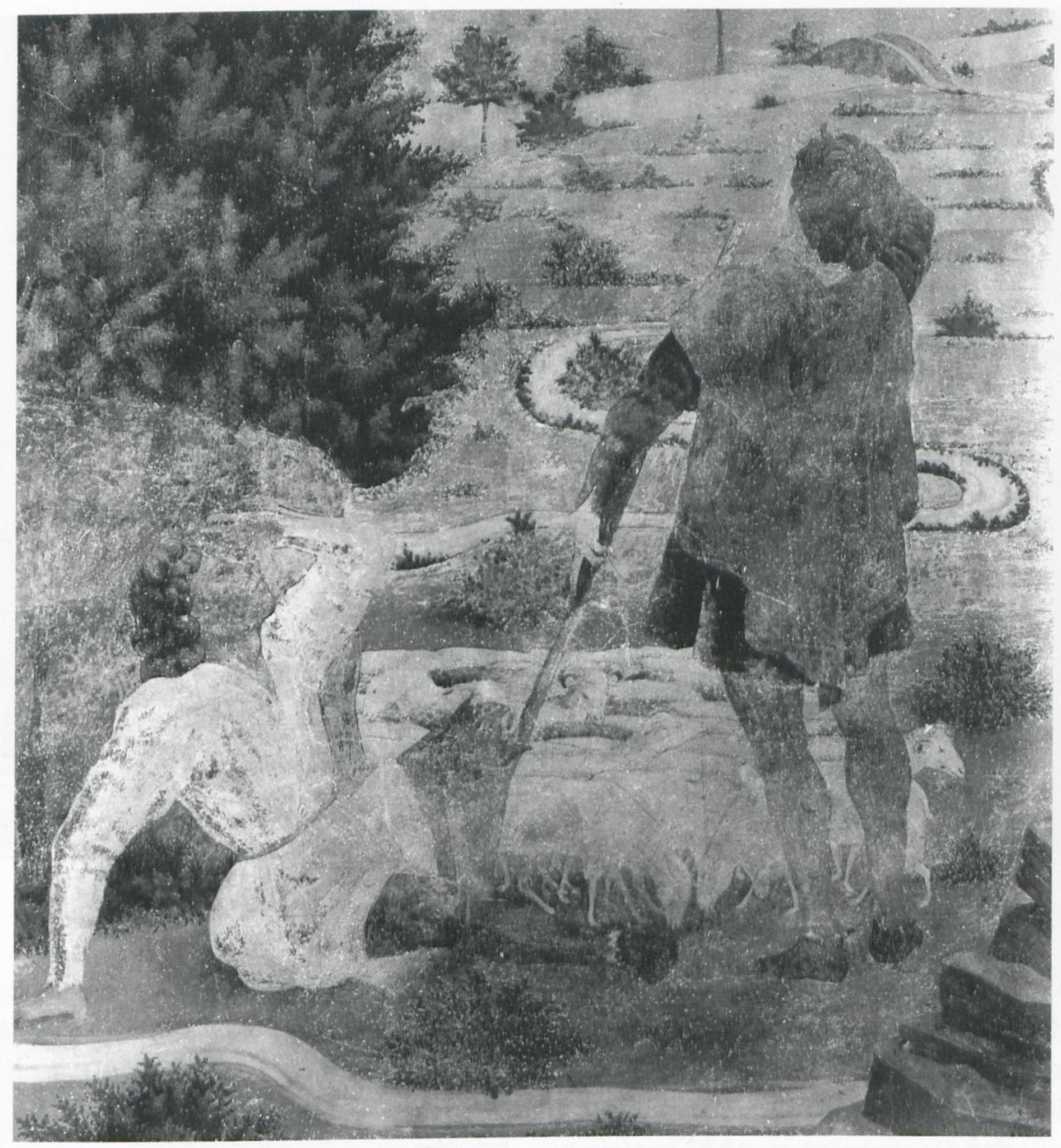

5 Baldovinetti, Fresko im Vorhof der Santissima Annunziata, Florenz, Detail: Hirten

dert ist - wie ja auch die Abzeichen des Großherzogtums Toskana, die Fortuna dem Medici-Herkules reicht, erst nach der Erhebung von Cosimo I. zum Großherzog (1569), nicht schon zu Giovios Zeiten vorgesehen werden konnten. ${ }^{53}$ Und wäre es nicht um 1520 politisch unklug gewesen, die starken republikanischen Kräfte in Florenz durch die mediceische Vereinnahmung des legendären Stadtgründers und „republikanischen“ Helden Herkules zu provozieren ? $^{54}$

Giovios Programm der Vertumnus-Lünette wirbt zwar für eine permanente Medici-Herrschaft, doch sehr subtil-versteckt: Das auf den ersten Blick ganz harmlose, aber mit dynastischen Anspielungen aufgeladene Bild unterscheidet sich so stark von der dick aufgetragenen Rhetorik der Herkules-Lünette, daß Giovio wohl kaum als Programmautor für letztere in Frage kommt. Außer- dem berichtet Allori, er habe das Fresko „tutto con gran inventione del Don Vincentio Borghini“ ausgeführt, was mindestens auf starke Abweichungen von Giovios Plan schließen läßt. ${ }^{55}$ Welches Programm aber könnte Giovio in logischem Zusammenhang mit der Vertumnus-Lünette ursprünglich für deren Pendant entworfen haben?

Die zwei erhaltenen Lünetten-Gesamtskizzen Pontormos beziehen sich wohl beide auf die südliche Lünette. ${ }^{56}$ Einige Passagen in Vasaris Viten erlauben jedoch Rückschlüsse auf Giovios Projekt für die heutige Herkules-Lünette. Vasari berichtet, daß Pontormo 1532 beauftragt wurde, die Ausmalung der Sala in Poggio a Caiano zu vollenden. ${ }^{57}$ Pontormo hatte 1520 oder 1521 den Auftrag für die Gestaltung beider Stirnseiten erhalten, ${ }^{58}$ das heißt, er hatte damals schon Giovios Programm für alle zwei Lünetten mitgeteilt bekommen. Otta- 


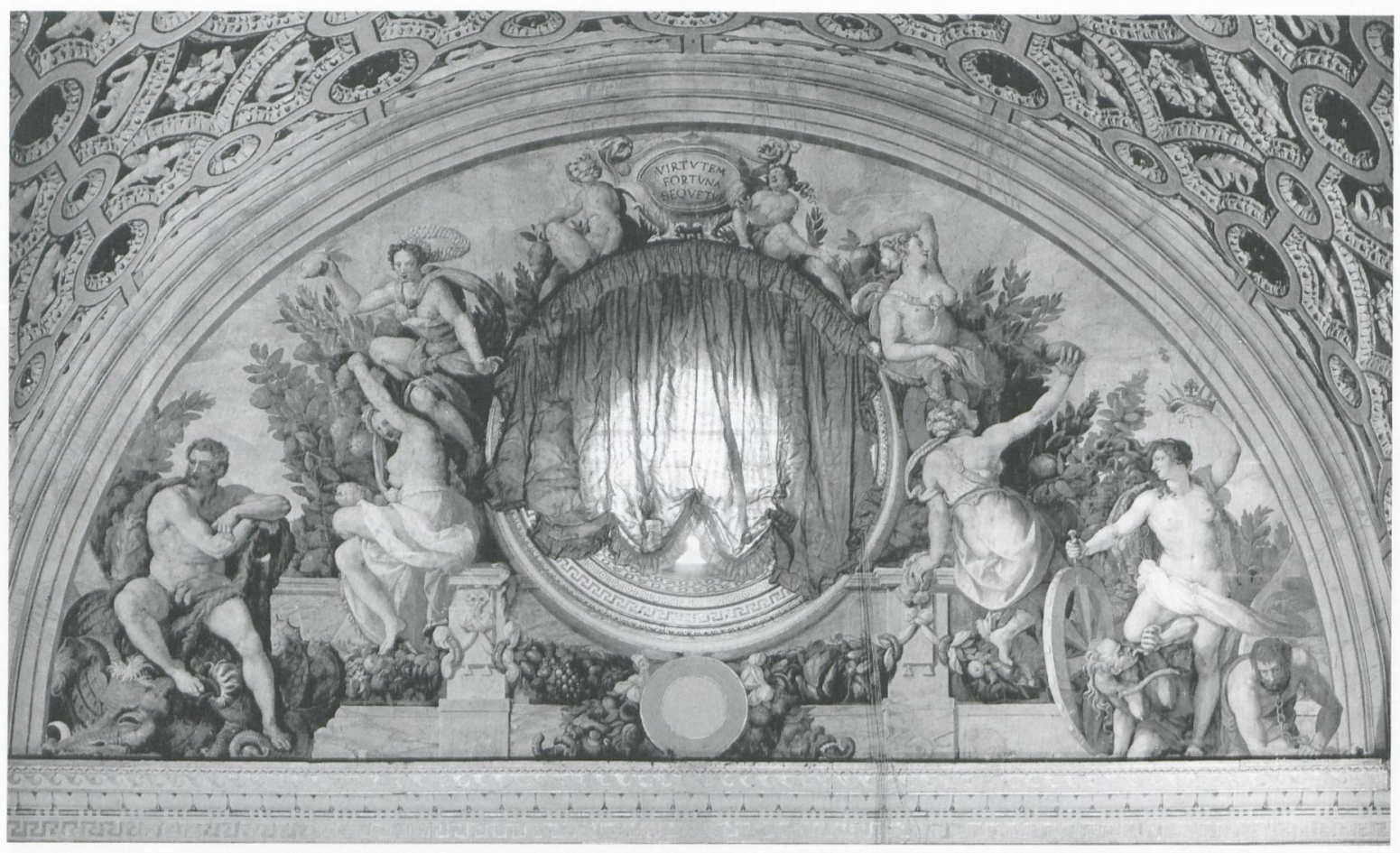

6 Allori, Lünettenfresko in der Sala grande (Nordseite), Villa Medici, Poggio a Caiano

viano und Giulio de' Medici (Clemens VII.) überwachten ab 1532 wie bereits 1519-1521 das Projekt, kannten also ebenfalls noch Giovios ursprüngliche Pläne. ${ }^{59}$ Die Wahrscheinlichkeit ist daher sehr groß, daß Pontormos ca. 1532 entstandene Zeichnungen Giovios originalen Programmentwurf reflektieren.

Laut Vasari lieferte Pontormo damals für Poggio a Caiano folgendes: „In uno de' quali cartoni, che sono oggi per la maggior parte in casa di Lodovico Capponi, è un Ercole che fa scoppiare Anteo, in un altro una Venere et Adone, et in una carta una storia d'ignudi che giuocano al calcio. “60 Da Vasari deutlich zwischen cartoni und carta unterscheidet, handelte es sich bei dem letztgenannten Thema der ignudi offenbar nur um eine kleine, nicht zur Ausführung bestimmte Zeichnung. ${ }^{61}$ Die beiden cartoni dürften nicht mit den großen Bildfeldern in der unteren Wandzone zusammenhängen, denn man wäre wohl kaum so inkonsequent gewesen, mythologische Szenen in den unvollendeten Zyklus antiker Historien gleichwertig zu integrieren. „Ercole che fa scoppiare Anteo" kommt eher für eine Sopraporta als für die Lünette in Frage, da Herkules mit Antäus, den er im Kampf hochhebt, eine kompakte Gruppe bildet. Die zweite von Vasari genannte Figurengruppe, Venus und Adonis, ließe sich dagegen analog zu Vertumnus und Pomona auf beide Seiten neben dem Okulusfenster in der Lünette verteilen. Das Liebespaar Venus und Adonis bildet außerdem ein thematisches Pendant zum Liebespaar Vertumnus und Pomona.

Zwei Zeichnungen vermitteln vielleicht eine Vorstellung von dem durch Vasari bezeugten, aber verlorenen Karton mit Venus und Adonis. Bereits 1956 sah Marcucci, gefolgt von Sinibaldi, Pontormos Anfang der 1530er Jahre zu datierende Zeichnung eines liegenden Jünglings (Abb. 7) als Vorstudie zum Adonis für die Lünette in Poggio a Caiano an. ${ }^{62}$ Dem läßt sich versuchsweise eine "Venus“ (Abb. 8) aus derselben Phase beigesellen. ${ }^{63}$ Beide Figuren sitzen bzw. liegen, könnten also wie in der Vertumnus-Lünette auf einer Mauerkomposition untergebracht werden. Der "Adonis“ ist in seiner Haltung fast ein Spiegelbild zu der rechts unter Pomona lagernden Frau (vgl. Abb. 3). Analog zu dieser wäre er mit den Füßen zum Okulusfenster hin (also links) anzuordnen; „Venus“ (auf der rechten Okulusseite gedacht) blickt nach links, also zu „Adonis“ hinüber. Ihre aus der Zeichnung heraus- 




7 Pontormo, „Adonis“ für Poggio a Caiano?, Florenz, Uffizien, 6630 F

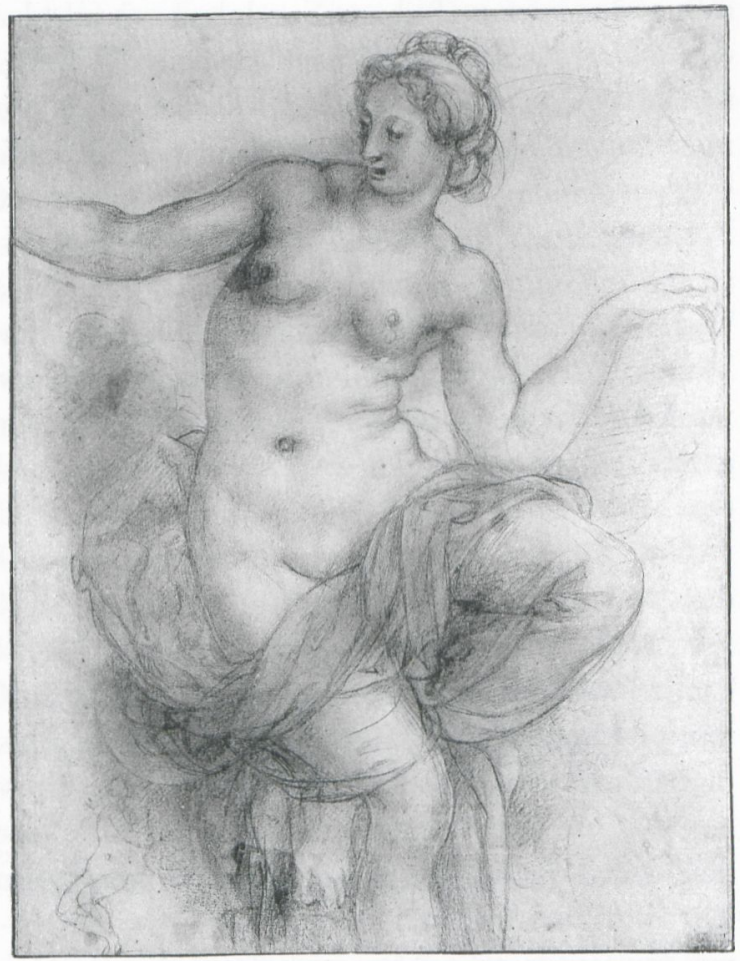

8 Pontormo, „Venus“ für Poggio a Caiano?, Hamburg, Kunsthalle, 21173 r greifende Geste könnte (ähnlich wie bei Pomona) einem Baum oder einer Frucht gelten.

Falciani hat die Hypothese aufgestellt, eine Venus-und-Adonis-Lünette sei 1531 als Bestandteil eines speziell auf Alessandro de' Medici zugeschnittenen Programms für die Sala in Poggio a Caiano konzipiert worden. Seine Interpretation baut auf Bonsignoris Ovid-Kommentar auf, dem zufolge Adonis für die Sünde der Luxuria steht. Falciani sieht in dem Umstand, daß Adonis versuchte, sich von seinem Laster durch die Jagd abzulenken, eine Parallele zu Pomonas Konzentration auf die Gartenarbeit, durch die sie lange keusch geblieben war. Da Alessandro de’ Medici oft für seine lussuria getadelt wurde, glaubt Falciani, die beiden Lünettenbilder hätten dem Herzog als positive Exempel dienen sollen, wie man durch körperliche Beschäftigung den Gedanken an das Laster verdrängt und die für das Regieren nötige Tugend erringt. ${ }^{64}$ Das überzeugt jedoch nicht: Die körperliche Betätigung hat Adonis den Tod auf der Jagd eingebracht, und Pomona wird in Poggio a Caiano ja gerade nicht für ihre Keuschheit, sondern für ihre Fruchtbarkeit gefeiert. 
Die Wahl des Themas „Venus und Adonis“ für die Lünette dürfte aus anderen Gründen erfolgt sein. Einen Deutungshinweis gibt der weit verbreitete Ovid-Kommentar das Raphael Regius, der die jährlichen Adonisfeste der Antike hervorhebt, die laut Ovid Venus selbst einrichtete. ${ }^{65}$ Nach Adonis' Tod soll Venus so traurig gewesen sein, daß sie einen Kompromiß erwirkte: Nur einen Teil des Jahres mußte Adonis fortan in der Unterwelt leben, danach durfte er zu Venus auf die Erde zurückkehren. Der antike Adoniskult feierte Tod und Auferstehung des Adonis als Sinnbild für das Absterben und Wiederaufleben der Pflanzenwelt im Jahreslauf. ${ }^{66}$

Berichte von Adonisfesten hat zum Beispiel Lukian in ,De Syria Dea' überliefert; daß der Adoniskult in der Renaissance gut bekannt war, belegt nicht nur der Kommentar von Regius, sondern auch die 1499 erschienene Hypnerotomachia Poliphili, in der das Adonisfest als abschließender Höhepunkt einer ganzen Reihe von Läuterungs- und Wiedergeburtsriten evoziert wird. ${ }^{67}$ Boccaccio und Piero Valeriano (der Astrologe Leos X., ${ }^{68}$ des Auftraggebers der Fresken in Poggio a Caiano) assoziierten in der Nachfolge von Macrobius ebenfalls Adonis mit dem Jahreszeitenwechsel. ${ }^{69}$

Ebenso wie Vertumnus und Pomona sind also auch Venus und Adonis eng mit dem Thema der Jahreszeiten und der pflanzlichen Regeneration verbunden und verweisen auf die Idee der zyklischen Wiederkehr, die ja das gesamte Programm in Poggio a Caiano bestimmt. Ich glaube allerdings, daß nicht nur diese Überlegungen zur Wahl des Themas Venus und Adonis geführt haben, sondern daß eine bestimmte literarische Quelle Giovios verlorenem Programm für die zweite Lünette von Poggio a Caiano zugrunde lag: Giovanni Pontanos ,De Hortis Hesperidum ‘ ${ }^{70}$

Giovanni Pontano war nicht nur ein hoch angesehener Literat, sondern auch Kanzler und wichtigster Berater des Königs von Neapel. Daher hatte er in diplomatischen Missionen öfters mit Lorenzo de’ Medici „Il Magnifico“ zu tun. ${ }^{71} 1493$ versuchte Pontano mit Lorenzos Sohn Piero ein Bündnis gegen Karl VIII. von Frankreich zustandezubringen, der Neapel erobern wollte. ${ }^{72}$ Natürlich tauschte man in jener Situation Höflichkeiten aus: Poliziano schmeichelte Pontano in einem Brief von 1494 mit der Sympathie, die sein Schüler Piero de’ Medici für Pontano empfinde, ${ }^{73}$ während Pontano seinerseits in den ab 1493 verfaßten Traktaten, De Liberalitate' bzw. ,De Magnificentia' Cosimo de' Medici als leuchtendes Vorbild rühmte. ${ }^{74}$

In diesem Zusammenhang wäre es möglich, daß ,De Hortis Hesperidum`von Pontano 1493 als ein Geschenk für den umworbenen Bündnispartner Piero de' Medici geschrieben wurde. ${ }^{75}$,De Hortis Hesperidum', ein von Vergils ,Georgica' inspiriertes Lehrgedicht über die Zitronenzucht, konnte zweifellos auf das Interesse der Medici rechnen, die mit Begeisterung Zitrusfrüchte zogen. ${ }^{76}$ Es soll im folgenden gezeigt werden, daß der Text zahlreiche Passagen enthält, die auf Medici-Symbolik sowie auf die schwierige politische Situation nach dem Tod des „Magnifico“ anzuspielen scheinen. Wie Bartolomeo Scalas ca. 1494/95 verfaßtes Werk ,De Arboribus' belegt, das für Lorenzo di Pierfrancesco de' Medici Propaganda machte, ${ }^{77}$ war es damals keineswegs ungewöhnlich, in einem landwirtschaftlichen Lehrgedicht politische Andeutungen unterzubringen.

Paolo Giovio, der Pontano sehr schätzte, charakterisierte dessen Stil unter anderem mit folgenden Worten: „Fu [...] di piacevole stile, \& di cortese favella: avenga ch'assai spesso fra le cose piu gravi, mescolava accortamente, \& con molta gentilezza di leggiadrissimi motti." "Mo „Motto“ ist bei Giovio ein Fachbegriff für den Textbestandteil einer Imprese. ${ }^{79}$ Für Giovio kennzeichnet es also Pontanos Texte, daß der Dichter in höfisch-schmeichelhafter Manier („cortese“, „con molta gentilezza“) Hinweise auf Impresen einstreute, versteckt zwischen ganz andersartigen Themen. Dies ist meines Erachtens auch bei ,De Hortis Hesperidum ' der Fall.

Um die Medici-Bezüge des Gedichtes zu verdeutlichen, muß zunächst kurz auf die mythologische Interpretation der sogenannten palle eingegangen werden: Die sechs Kugeln auf dem MediciWappen konnten nämlich als Äpfel der Hesperiden aufgefaßt werden. ${ }^{80}$ Diese Gleichsetzung beruhte auf einem Wortspiel: „Äpfel der Hesperiden " wurde damals oft als poetisches Synonym für Zitrusfrüchte gebraucht, und diese wiederum hatten den lateinischen Namen mala medica. ${ }^{81}$ "Medica" bedeutet "aus dem Reich der Meder" (aus Persien), ließ sich aber natürlich auch auf die Me- 
dici beziehen, zumal Zitronen in der Medizin eingesetzt wurden. ${ }^{82}$

Seit etwa 1438 konnten Orangen (mala aurea) stellvertretend für die roten palle des Medici-Wappens stehen. ${ }^{83}$ Mala aurea erscheinen im Quattrocento als Schmuck in Medici-Handschriften, und die auffälligen Orangen in einigen von den Medici bestellten Gemälden sind wohl als heraldische Allusion auf die Auftraggeber zu deuten. ${ }^{84}$ Der deutsche Gelehrte Reuchlin sah an den Decken des Florentiner Medici-Palastes „Hesperidum hortos ac aurea mala “. ${ }^{85}$ In Medici-Panegyriken der Zeit wurde öfters auf die Hesperiden angespielt. ${ }^{86}$ Der Vergleich der palle mit den Hesperidenäpfeln war vermutlich deswegen beliebt, weil die goldenen Äpfel als Zeichen für Tugend und Unsterblichkeit galten. ${ }^{87}$

In der mythologischen Einleitung von ,De Hortis Hesperidum` erzählt Pontano, wie Venus ihren toten Geliebten Adonis in den Hesperidenbaum verwandelt. Diese Metamorphose besitzt kein Vorbild in der antiken Literatur: Laut Ovid verwandelte sich Adonis in eine Blume, während die Entstehung des Hesperidenbaumes als Hochzeitsgeschenk der Erde an Zeus erklärt wird, ohne daß Adonis daran beteiligt gewesen wäre. ${ }^{88}$ Bei Pontano erhält Venus die Anregung zur Verwandlung des Adonis vom Lorbeer, der ebenfalls durch eine Metamorphose entstand. Auffälligerweise wird in dem in Minuskeln gesetzten Gedicht „Lauro“ (Lorbeer) wie ein Personenname groß geschrieben, was in der Medici-Panegyrik der Standard-Hinweis auf Lorenzo il Magnifico ist. ${ }^{89}$ Pontano beschreibt den Hesperidenbaum als eine dem Lorbeer gleichende Pflanze, die goldene Äpfel trage. Diese Verbindung von Lorbeer (Lorenzo) und Hesperidenbaum (mit goldenen Äpfeln, d. h. mit Medici-palle) war in der Medici-Panegyrik gebräuchlich: Beispielsweise schrieb Vitellius, der Medici-Baum werde dem der Hesperiden vorgezogen und trage goldene Äpfel; bei Pollastrino verwandelt sich Florentia in einen Lorbeerbaum, dessen Früchte mit den Hesperidenäpfeln wetteifern. ${ }^{90}$

In diesem Kontext erscheint es bedeutungsvoll, daß Pontano mit Bezug auf die Fruchtbarkeit des Hesperidenbaumes viermal in ganz betonter Stellung das Wort „semper“ präsentiert („flore novo semper, semper quoque foetibus aucta" bzw. „semper flore novo, semperque ornabere pomis“): eine Anspielung auf das bekannte Medici-Motto „semper"?" Hat Pontano die ungewöhnliche Adonis-Metamorphose, die sich bewußt von den antiken Quellen absetzt, nur deswegen erfunden, um den Medici-Lorbeer, Topoi der Medici-Panegyrik, ein Medici-Motto und die palle des MediciWappens effektvoll in der Einleitung unterzubringen?

Anschließend berichtet Pontano, daß Herkules die Hesperidenäpfel errang und sie nach Italien brachte, wo fortan die Zitrusfrüchte heimisch wurden. Wenig später tritt Juno auf, die aus Wut das Geschlecht der Hesperidenbäume fast vernichtet. Doch Venus sammelt im Wald der Meder die Überreste, bringt sie nach Italien und belebt die Pflanze dort wieder. In Zukunft, so Pontano, solle der Baum immerfort bestehen bleiben und in „althergebrachter Verehrung" durch die Nachkommen gepflegt werden, „dem Volk der Äneaden zum Nutzen“. Diese Regeneration einer schon beinahe abgestorbenen, verehrungswürdigen „Medici“Pflanze durch göttliche Hilfe bildet eine verblüffende Parallele zu Ariosts Bitte um überirdischen Beistand für den kranken Medici-Lorbeer in dem Gedicht, das der Vertumnus-und-Pomona-Lünette zugrundeliegt.

Das Absterben der Hesperidenbäume in ,De Hortis Hesperidum' läßt sich als Anspielung auf den Tod von Lorenzo il Magnifico (1492) verstehen, den Pontano als einen bedrohlich destabilisierenden politischen Faktor beklagt hatte; ${ }^{92}$ die Wiederbelebung des Gewächses wäre dann ein optimistischer Ausblick auf die Regeneration der Dynastie unter Lorenzos Sohn Piero. Der darauf folgende Ratschlag an den Gärtner, wie man die Zitruspflanze vor gefährlichem Klima schützen könne, paraphrasiert eine Imprese Pieros ${ }^{93}$ und kommt zu dem beruhigenden Schluß: „So wirst du Hitze und Kälte besiegen“.

Die Frage, ob Pontano ,De Hortis Hesperidum ‘ tatsächlich als Medici-Panegyrik verfaßte, mag offenbleiben $^{94}$ - fest steht jedenfalls, daß das Gedicht eine Reihe von Motiven enthält, die ausgesprochen gut in die Gedankenwelt der Medici und in den Programmkontext von Poggio a Caiano paßten. Giovios Verehrung für Pontano läßt sich 
genau in jene Jahre zurückverfolgen, in denen er das Bildprogramm für die Villa entwarf: 1521 berichtet er in einem Brief, er habe in seinen Räumen im Palazzo Medici eine Sammlung von Bildnissen bedeutender Männer aufgestellt, und an allererster Stelle seiner Aufzählung von 16 Portraits nennt er Pontano. ${ }^{95}$ Überdies kannte Giovio ,De Hortis Hesperidum ' nicht nur, sondern zählte diese „versi divini“ in warmen Lobesworten zu Pontanos gelungensten, „sublimen“ Dichtungen. ${ }^{96} \mathrm{Da}$ Giovio nach eigener Aussage bei Pontano mit geschärftem Blick auf versteckte Motti bzw. Impresen achtete, ${ }^{97}$ werden ihm die (absichtlichen oder unabsichtlichen) Medici-Bezüge dieses Gedichtes geradezu entgegengesprungen sein.

,De Hortis Hesperidum' bot sich Giovio als perfekte Programmgrundlage für die zweite Lünette in Poggio a Caiano an. Wie Ariosts, Ne la Stagion' basiert Pontanos Gedicht auf Vergils, Georgica', was für die Dekoration eines Landhauses besonders angemessen ist. Venus und Adonis, die Protagonisten des Gedichts, bilden als Liebespaar ein geeignetes Pendant zu Vertumnus und Pomona und können ebenso wie diese die zyklische Wiederkehr der Zeit ausdrücken. Die von Pontano hergestellte Verbindung zwischen Venus, Adonis und dem Hesperidenbaum erlaubt die Darstellung der Hesperidenäpfel als palle, analog zu der dominanten Rolle des Lorbeers in der Vertumnus-undPomona-Lünette. Sowohl goldene Äpfel als auch Lorbeer sind aus Wappen bzw. Impresen und Gedichten wohlbekannte Medici-Zeichen und stehen außerdem traditionell für virtus und gloria. Das neue Ausschlagen des Lorbeers bzw. die Wiederbelebung des hesperidischen Zitronenbaumes spielen auf die Regeneration der Medici-Dynastie an. Gleichzeitig signalisieren die beiden immergrünen Pflanzen den erhofften ewigen Bestand der Familie. ${ }^{98}$ Als Gegenstück zu den Jahreszeitenpersonifikationen, die Vertumnus und Pomona Gesellschaft leisten, kommen in der Venus-und-Adonis-Lünette die Horen in Betracht: Sie sind laut Homer und Polizian die Begleiterinnen der Venus und symbolisieren den Zeitzyklus, bei Philostrat auch den Jahreslauf. ${ }^{99}$

Venus galt in der Antike (z. B. bei Plinius und Festus) als Beschützerin der Gärten, als „venus hortorum“. In dieser Eigenschaft wurde sie von
Varro, Lukrez und Columella in Gedichten über die Natur sowie in Ovids ,Fasti‘ angerufen. ${ }^{100} \mathrm{Ve}-$ nus ist die Frühlingsbringerin, die neues Wachstum in der Natur auslöst - ein schon durch Poliziano und Botticelli in die Medici-Kunst eingeführtes Thema. ${ }^{101}$ Ohne sie gedeiht nichts; und so wie Vertumnus und Pomona dem kranken Lorbeer zur Hilfe kommen, ist es in Pontanos ,De Hortis Hesperidum 'Venus hortorum als Beschützerin der Gärten, die die Hesperidenpflanze vor der völligen Vernichtung bewahrt und wiederbelebt. Venus zählt also an prominenter Stelle zu „diique deaeque omnes studium quibus arva tueri“. Dieser Vers aus Vergils, Georgica' sollte komplett über der Vertumnus-Lünette stehen, wie ein bei der jüngsten Restaurierung entdeckter, in den Putz geritzter Schriftzug beweist. ${ }^{102}$ Schließlich wurde aber nur die zweite Hälfte des Relativsatzes („studium quibus arva tueri“") dort angebracht. Die Vermutung liegt nahe, daß man zunächst für jede der beiden Lünetten ein eigenes Motto aus den, Georgica' vorgesehen hatte, ${ }^{103}$ in einem zweiten Planungsschritt aber beschloß, den genannten Relativsatz auf zwei Lünetten aufzuteilen, dadurch die enge thematische Zusammengehörigkeit der beiden Fresken noch betonend: Venus und Adonis, Vertumnus und Pomona konstituieren gemeinsam die „Götter und Göttinnen alle, deren Aufgabe die Pflege der Bäume ist".

Der Venusgarten versinnbildlicht oft das Goldene Zeitalter. Das liegt daran, daß das Goldene Zeitalter eher zeitlich als räumlich definiert ist: Es ist ein paradiesischer Zustand zu Anfang des Zeitzyklus', dadurch der Jugend und dem Frühling vergleichbar. ${ }^{104}$ So ergibt sich eine Beziehung zu Venus, der mit Jugend und Frühling assoziierten Göttin; im Venusreich herrscht ebenso wie im Goldenen Zeitalter ewiger Frühling. ${ }^{105}$ Das Venusreich wiederum hängt mit dem Hesperidengarten zusammen, da die Hesperidenäpfel der Venus geweiht sind ${ }^{106}$ - deswegen erwähnt Poliziano in seiner Beschreibung des Venusgartens einen Baum mit goldenen Äpfeln. ${ }^{107}$ Auch wegen ihrer Farbe und wegen der Eigenschaft, ewige Jugend zu verleihen, paßten die Hesperidenäpfel in das Bild des Goldenen Zeitalters. ${ }^{108}$

Ein Gemälde wie Botticellis ,Primavera', das Venus in einem hesperidischen palle-Orangenhain 
zeigt, ist als Versprechen eines Goldenen Zeitalters für Florenz interpretiert worden. ${ }^{109}$ Eine ähnliche Deutung bietet sich in Poggio a Caiano an. Während die Vertumnus-Lünette einen arkadischen Garten vor Augen führt, in dem „Glovis“ und das Wiederaustreiben des Lorbeers die Hoffnung auf eine Rückkehr des saeculum aureum ausdrücken, erfüllt sich diese Hoffnung im Venusreich der gegenüberliegenden Lünette. Der Zeitzyklus mündet in einen ewigen paradiesischen Frühling. Pontano hatte der Hesperiden-Dynastie ein immerwährendes Bestehen gewünscht; daher könnte im Venus-Fresko analog zur „Glovis“-Imprese die Anbringung des Medici-Mottos „semper" geplant gewesen sein, das so betont und gehäuft in Pontanos Gedicht auftaucht und das bereits bei früherer Gelegenheit mit "Glovis“ gepaart worden war. ${ }^{110}$

Einiges spricht dafür, daß das unausgeführte Lünettenprogramm nicht in Vergessenheit geriet. Pontormos Venus-und-Adonis-Karton konnte schließlich, wie Vasari schreibt, im Haus des Lodovico Capponi besichtigt werden und so eine Nachwirkung entfalten. ${ }^{111} \mathrm{Daß} 1566$ in einem (wohl von Vincenzo Borghini organisierten $)^{112}$ „Triumphzug der Venus“ Adonis von Putten mit Körben voller goldener Äpfel eskortiert wurde, ${ }^{113}$ reflektiert offenbar die nur von Pontano hergestellte Verbindung zwischen Adonis und den Hesperidenäpfeln. Borghini, der 1578 das Thema für die fehlende Lünette in Poggio a Caiano festlegte, dürfte als guter Bekannter von Pontormo sowie durch Giovios Freund Vasari Kenntnis von Giovios ursprünglichem Konzept besessen haben. ${ }^{114}$ Bezeichnenderweise malte Allori die palle in der Lünette als $\mathrm{Zi}$ tronen und nicht (wie sonst in der Medici-Kunst üblich) als Orangen: eine Spätfolge von ,De Hortis Hesperidum', worin es ausdrücklich um Zitronenbäume geht?

Borghini scheint in Poggio a Caiano nicht aus Unwissenheit, sondern mit Absicht Giovios concetto abgewandelt zu haben: Er behielt den Hesperidengarten als Schauplatz bei, ersetzte aber Venus und Adonis durch die konventionellere, eindeutiger propagandistische, leichter verständliche Herkules-Fortuna-Allegorie. Diese enthält dieselbe Idee vom Stillstand des Zeitzyklus' und vom Goldenen Medici-Zeitalter, die Giovio in Anlehnung an Pontano subtil-verschlüsselter zum Ausdruck bringen konnte. ${ }^{115}$

Neben der allgemeinen Botschaft von Wachsen und Bestand der Medici-Dynastie, die jeder künftige Herr der Villa auf sich beziehen konnte, sollten die beiden Lünetten in Poggio a Caiano vermutlich noch eine speziellere, auf den Auftraggeber Leo X. bezogene Aussage enthalten. Wie Vasari berichtet, ließ Leo X. den Salone in Poggio a Caiano zu Ehren seines Vaters Lorenzo il Magnifico ausmalen. ${ }^{116}$ Das bedeutet aber nicht, daß der Papst nicht auch sich selbst damit ein Monument errichtet hätte: Sein Wappen beherrscht das Zentrum des Gewölbes. ${ }^{117}$ Kliemann und Winner haben bereits herausgestrichen, daß sich in den Wandbildern des Salone Anspielungen auf die leoninische Gegenwart finden; so huldigt etwa Andrea del Sartos ,Tribut an Caesar' gleichzeitig Lorenzo und Leo und gibt zu verstehen, daß der Sohn als würdiger Nachfolger die Machtstellung des Vaters noch ausgebaut hat. ${ }^{118}$ Diese Vater-Sohn-Relation scheint mir auch in den beiden Lünetten thematisiert, wie im folgenden knapp skizziert werden soll.

Für Giovio hatte es nahe gelegen, dem Lorbeerhain der Vertumnus-Lünette den Garten der Hesperiden gegenüberzustellen, da Leo X. im Vatikan einen mit Orangenbäumen und einer Venusstatue geschmückten Skulpturenhof besaß, den Fulvio und Castiglione (wohl in Anspielung auf die Medici-palle) als Hesperidengarten interpretierten. ${ }^{119}$ Ein Baum mit palle war schon bei Leos Possesso zu sehen gewesen; goldene Äpfel begegneten in der Leo-Panegyrik und bei einer Orangenschlacht, die für den Papst aufgeführt wurde. ${ }^{120}$ Der päpstliche Günstling Valeriano stellte sogar eine Verbindung zwischen Leos Schicksal und dem Gedeihen der mediceischen Zitronenbäume her. ${ }^{121}$ Giovio, der seit 1517 am Hof von Leos engstem Vertrauten Giulio de' Medici lebte, ${ }^{122}$ wußte natürlich von der Bedeutung der goldenen Äpfel in der leoninischen Propaganda. Der Hesperidengarten bot ein geeignetes Bild für das goldene Friedensreich, das Leo $\mathrm{zu}$ errichten versprach, ${ }^{123}$ und die medizinisch nutzbaren „mala medica" konnten auf das heilsame Wirken des Medici-Papstes bezogen werden, der sich zum "christus medicus “ stilisierte. ${ }^{124}$

Lorenzo il Magnifico hatte sich als erster Medici eine Lorbeerimprese erwählt, und die Wiederbele- 
bung der Lorbeersymbolik zur Zeit Leos geschah ausdrücklich in Erinnerung an den glorreichen Lorenzo. ${ }^{125}$ Das Vertumnus-Fresko spricht die beiden großen Themen an, die in unzähligen Gedichten des Magnifico-Kreises formuliert worden waren: die Verherrlichung von Lauro-Lorenzo und die Feier eines Medici-Arkadiens. Auf diesen „lorenzianischen" Lorbeerhain sollten in der zweiten Lünette die „mala medica“ Leos antworten, um den Sohn als Vollender des Werks seines Vaters zu präsentieren: Unter dem Zeichen des friedensstiftenden, gerechten Medici-Papstes kehrt das Goldene Zeitalter zurück, nach dem sich die Arkadier der Lorenzo-Zeit nostalgisch gesehnt hatten.

Abschließend seien noch einmal kurz die wichtigsten Thesen resümiert. Durch die Annahme, das Programmkonzept für die Nordlünette basiere auf der von Giovio sehr geschätzten Dichtung ,De Hortis Hesperidum', läßt sich erklären, warum Pontormo laut Auskunft Vasaris gerade einen Karton mit Venus und Adonis für Poggio a Caiano schuf: Pontanos Gedicht, möglicherweise für Piero de' Medici geschrieben, erzählt eine neu erfundene Legende von Venus und Adonis, die eine verblüffende Parallele zu Ariosts ,Ne la Stagion“ bildet. So wie in diesem Werk Ariosts, das der Vertumnus-und-Pomona-Lünette zugrundeliegt, Pomona für die Wiederbelebung des kränkelnden Medici-Lorbeers sorgen soll, so rettet bei Pontano

\section{Anmerkungen}

Der vorliegende Aufsatz ging aus einem Referat hervor, das ich 1994 bei einem Studienkurs der Bibliotheca Hertziana („Kunst und Kunstpolitik am Hofe Leos X.“) vortrug. Ich danke Herrn Prof. Dr. Matthias Winner, PD Dr. Julian Kliemann, Dr. Michael Rohlmann und Dr. Tobias Leuker für die kritische Lektüre des Manuskripts. Für altphilologischen Beistand bin ich Dr. Reinhard Kratz zu großem Dank verpflichtet.

1 Erkinger Schwarzenberg, Glovis, Impresa di Giuliano de' Medici, in: Mitteilungen des Kunsthistorischen Institutes in Florenz, 39, 1995, 140-166; zur Kloakeninschrift: 153-155, fig. 13.

2 Die Autoren der im folgenden zitierten grundle-
Venus hortorum den bedrohten Hesperidenbaum, eine weitere „Medici-Pflanze“. Ebenso wie das Liebespaar Vertumnus und Pomona symbolisieren auch Venus und Adonis den Kreislauf des Jahres. Während aber die Vertumnus-Lünette ein mediceisches Arkadien präsentiert (dessen Herrscher Pan bei der jüngsten Restaurierung neu „entdeckt “ wurde), konnte der Venusgarten als Sinnbild des Goldenen Zeitalters verstanden werden und damit als Erfüllung des von den Arkadiern Ersehnten gelten.

Beachtet man die Rolle von Lorbeer bzw. Hesperidenäpfeln in der Selbstdarstellung von Lorenzo il Magnifico bzw. Leo X. de' Medici, so erscheint die Vertumnus-Lünette als Hommage an den Vater des Auftraggebers, der dann mit der Venus-und-Adonis-Lünette triumphal das von Leo $\mathrm{X}$. herbeigeführte Goldene Zeitalter gegenüberstehen sollte. Die doppelte Regeneration einer „Medici-Pflanze" verkündete die Hoffnung der Familie, aus schwierigen Situationen stets gestärkt hervorzugehen - eine begründete Hoffnung, wie die Schicksale von Lorenzo und Leo eindrucksvoll bewiesen. So, wie nach dem Tod des Magnifico und dem darauf folgenden langjährigen Medici-Exil ein Mitglied der Familie zu den glanzvollsten päpstlichen Ehren emporgestiegen war, so würde sich Fortuna den Medici immer wieder zuwenden - womit sich der Kreis schließt, der zum Ausgangspunkt dieser Überlegungen zurückkehrt: Glovis. genden Publikationen zum Thema mögen mir die überaus verknappte Zusammenfassung verzeihen. Matthias Winner, Pontormo und die Medici in Poggio a Caiano, in: Sitzungsberichte. Kunstgeschichtliche Gesellschaft zu Berlin, N. F., Heft 12, Okt. 1963 - Mai 1964, 9-11. Id., Cosimo il Vecchio als Cicero. Humanistisches in Franciabigios Fresko zu Poggio a Caiano, in: Zeitschrift für Kunstgeschichte, 33, 1970, 261-297. Id., Pontormos Fresko in Poggio a Caiano. Hinweise zu seiner Deutung, in: Zeitschrift für Kunstgeschichte, 35, 1972, 153197. Julian Kliemann, Vertumnus und Pomona. Zum Programm von Pontormos Fresken in Poggio a Caiano, in: Mitteilungen des Kunsthistorischen 
Institutes in Florenz, 16, 1972, 293-328. Id., Politische und humanistische Ideen der Medici in der Villa Poggio a Caiano. Untersuchungen zu den Fresken der Sala grande, Diss. Heidelberg 1976. Id., Il Pensiero di Paolo Giovio nelle pitture eseguite sulle sue „invenzioni“, in: Paolo Giovio. Il Rinascimento e la memoria. Atti del Convegno (Como, 3.-5.6.1983), Como 1985, 197-222. Id., Gesta dipinte. La grande decorazione nelle dimore italiane dal Quattrocento al Seicento, Mailand 1993, 35-36. Janet Cox-Rearick, Dynasty and Destiny in Medici Art. Pontormo, Leo X, and the two Cosimos, Princeton 1984.

3 Julian Kliemann, Rezension zu Cox-Rearicks „Dynasty and Destiny in Medici Art“, in: Kunstchronik, 39, 1986, 132-141, hier: 135.

4 Winner 1972 (wie Anm. 2), 177s. Kliemann 1972 (wie Anm. 2), 304-306.

5 Kliemann 1976 (wie Anm. 2), 47-58.

6 Vgl. auch Gerhart B. Ladner, Vegetation Symbolism and the Concept of Renaissance, in: De Artibus Opuscula XL. Essays in honor of Erwin Panofsky, hg. von Millard Meiss, I, New York 1961, 303-322.

$7 \quad$ Kliemann 1972 (wie Anm. 2), 322s.

8 Wie die Restaurierung der Lünette ergab, übermalte erst Allori die Mauer mit einem Himmelsausblick. Vgl. A. Manetti Piccinini, Pontormo svisato dall'Allori, in: Il Giornale dell'Arte, 10, 1993, N. $113,42$.

9 Winner 1963/64 (wie Anm. 2), 9. Winner 1972 (wie Anm. 2), 159. Kliemann 1972 (wie Anm. 2), 306.

10 Le Vite de' più eccellenti Pittori Scultori ed Architettori scritte da Giorgio Vasari pittore aretino con nuove annotazioni e commenti di Gaetano Milanesi, 7 Bde., Florenz 1878-1881, VI, 265.

11 Kliemann 1976 (wie Anm. 2), 46.

12 Ebd., 43s. Thomas Da Costa Kaufmann, Arcimboldo and Propertius. A Classical Source for Rudolf II as Vertumnus, in: Zeitschrift für Kunstgeschichte, 48, 1985, 117-123, hier: 118-120.

13 Kliemann 1972 (wie Anm. 2), 297-303. Luciano Berti, L'opera completa del Pontormo, Mailand 1973, 96. Kliemann 1976 (wie Anm. 2), 40-46. CoxRearick (wie Anm. 2), 122. Salvatore S. Nigro, Pontormo. Zeichnungen, München u.a. 1991, Kat. Nr. 24. Litta Maria Medri, Il mito di Lorenzo il Magnifico nelle decorazioni della villa di Poggio a Caiano, Florenz 1992, 79. Sheryl E. Reiss, Cardinal Giulio de' Medici as a patron of art, 1513-1523, Diss. Princeton University 1992, 479. Luciano Berti, Pontormo e il suo tempo, Florenz 1993, 214. Litta Medri, Vertumno e Pomona di Poggio a Caiano, in: Il Pontormo. Le opere di Empoli, Carmignano e Poggio a Caiano, Venezia 1994, 50-66, hier: 63. Salvatore S. Nigro, Pontormo, Mailand 1994, Kat.
Nr. VII. Philippe Costamagna, Pontormo, Mailand 1994, 155. Anna Forlani Tempesti / Alessandra Giovannetti, Pontormo, Florenz 1994, $118 f$.

14 Ovid, Metamorphosen, XIV, 765ss. Carlo Falciani, Alcuni artisti alla villa del Poggio, in: Artista, 1992, 112-125, hier: 116. Id., Pontormo. Disegni degli Uffizi (Gabinetto Disegni e Stampe degli Uffizi, 79), Florenz 1996, 95. Vgl. auch Alessandro Conti, Pontormo, Como 1995, 31-34 (sieht die Figuren als „benandanti“).

15 Litta Maria Medri, Pontormo a Poggio a Caiano, Florenz 1995, 13, 21. Medri plädiert für die „Kompatibilität" beider Interpretationen: „Pan giunge ad identificarsi con Vertumno".

16 Winner 1972 (wie Anm. 2), 165. Aufgrund dieses Attributes hatte bereits Winner den Alten als „Pan“ angesprochen.

17 Paulys Realencyclopädie der classischen Altertumswissenschaft, 12. Halbband, Stuttgart 1909, Sp. 2055-2070, und Supplementband VIII, Stuttgart 1956, Sp. 1005.

18 Giovanni Boccaccio, L'Ameto o Commedia delle Ninfe Fiorentine, in: id., Opere, hg. von Nicola Bruscoli, V, Bari 1940. Über Pomonas Garten: 6775, hier: 70 .

19 Kliemann 1976 (wie Anm. 2), Anhang 40.

20 Schon wegen dieser anstößigen Posen halte ich es für ausgeschlossen, daß in den beiden Figuren neben dem Okulus Lorenzo Duca d'Urbino und Maria Salviati portraitiert sein sollen, wie Medri 1994 (wie Anm. 13, 60s) vorgeschlagen hat.

21 Kliemann 1976 (wie Anm. 2), 62.

22 Andere Beispiele für die Anordnung der Jahreszeiten von rechts nach links bei Cox-Rearick (wie Anm. 2), 203.

23 Der römische Pan (Faunus) hatte sein Fest, die Lupercalien, am 15.2.; vom Charakter des Festes (Reinigung $=$ februatio) leitet jener Wintermonat seinen Namen ab. Vgl. Anm. 17.

24 Pauly (wie Anm. 17), 3. Halbband, Stuttgart 1895, Sp. 1342-1344.

25 Vasari / Milanesi (wie Anm. 10), VI, 264s: „si mise con tanta diligenza a studiare, che fu troppa; percioché guastando e rifacendo oggi quello che avea fatto ieri, si travagliava di maniera il cervello, che era una compassione". Zu den Vorzeichnungen siehe Janet Cox-Rearick, The Drawings of Pontormo. A Catalogue Raisonné with Notes on the Paintings, 2 Bde., New York ${ }^{2} 1981$, I, 172-190. Ead., Aggiunte al "Corpus" dei Disegni del Pontormo: 1981-1994, in: Pontormo e Rosso. Atti del Convegno di Empoli e Volterra. Progetto Appiani di Piombino, hg. von Roberto P. Ciardi / Antonio Natali, Venezia 1996, 62-68, hier: 63, 200, Abb. 3436.

26 Cox-Rearick 1981 (wie Anm. 25), Cat. 135, Abb. 
127 (Uffizi 6515 F r); Cat. 136, Abb. 128 (Uffizi 6685 F r); Cat. 137, Abb. 129 (Uffizi 6599 F r).

27 Baldovinettis Fresko befindet sich im Chiostrino dei Voti der Chiesa della Santissima Annunziata in Florenz. Pontormo steuerte 1514-1516 ebenfalls ein Wandbild zur Dekoration des Chiostrino bei, war damals also ständig mit Baldovinettis Werk konfrontiert. Vgl. Costamagna 1994 (wie Anm. 13), 120ss.

28 Rudolf Preimesberger, Obeliscus Pamphilius. Beiträge zu Vorgeschichte und Ikonographie des Vierströmebrunnens auf Piazza Navona, in: Münchner Jahrbuch der bildenden Kunst, 3. Folge, 25, 1974, 77-162, hier: 132. Id., Eine Peripetie in Stein? Bemerkungen zu Alessandro Algardis Relief der Begegnung Leos des Großen mit Attila, in: Festschrift für Hermann Fillitz zum 70. Geburtstag (Aachener Kunstblätter, 60), Köln 1994, 397-416, hier: 410.

29 Detailabbildungen bei Medri 1995 (wie Anm. 15), 32, 33.

30 Jonas blickt mit schräg zurückgelehntem Oberkörper zu Gottvater an der Decke der Sixtinischen Kapelle auf; im Gegensatz zum heidnischen Gott Vertumnus darf der Prophet den Höchsten allerdings unverhüllt schauen. Vgl. Matthias Winner, Giona: il linguaggio del corpo, in: La Cappella Sistina. La volta restaurata: il trionfo del colore, Novara 1992, 110-119, hier: 116 und Abb. S. 117. Michael Rohlmann, Michelangelos "Jonas“. Zum Programm der Sixtinischen Decke, Weimar 1995, 34. Zu Pontormos Michelangelo-Zitaten in Poggio a Caiano siehe Janet Cox Rearick, The Drawings of Pontormo, Cambridge 1964, 41s. Kathleen Weil-Garris Posner, Comments on the Medici Chapel and Pontormo's Lunette at Poggio a Caiano, in: The Burlington Magazine, 115, 1973, 641-649. Forlani Tempesti / Giovannetti (wie Anm. 13), 119.

31 Zur Lichtführung in Pontormos Fresko siehe die zahlreichen Detailabbildungen bei Silvestro Bardazzi / Eugenio Castellani, La villa medicea di Poggio a Caiano, II, Prato 1981. Zu natürlichen und fiktiven Lichtquellen in der Renaissancemalerei vgl. Cecil Gould, On the Direction of Light in Italian Renaissance Frescoes and Altarpieces, in: Gazette des Beaux-Arts, 97, 1981, 21-25. Martin Kemp, In the light of Dante. Meditations on natural and divine light in Piero della Francesca, Raphael and Michelangelo, in: Ars naturam adiuvans. Festschrift für Matthias Winner zum 11. März 1996, hg. von Victoria v. Flemming / Sebastian Schütze, Mainz 1996, 160-177.

32 Jacopo Nardi, I sette trionfi del secol d'oro Facti dalla Compagnia del Bronchone l'anno MDXII (abgedruckt bei Kliemann 1976, wie Anm. 2, 230ss). Die Idee der „Divina Provvidenza“ betont bei David Roy Wright, The Medici Villa at Olmo a
Castello: Its History and Iconography, Diss. Princeton 1976, 234-236.

33 Erwin Panofsky, Et in Arcadia Ego: Poussin and the Elegiac Tradition, in: id., Meaning in the visual arts. Papers in and on Art History, Garden City 1955, 295-320, hier: 297-304.

34 Hans Henrik Brummer, Pan Platonicus, in: Konsthistorisk Tidskrift, 33, 1964, 55-67, hier: 58.

35 Carol Kidwell, Sannazaro and Arcadia, London 1993, 9, 11, 29s.

36 André Chastel, Art et humanisme à Florence au temps de Laurent le Magnifique. Etudes sur la Renaissance et l'humanisme platonicien, Paris ${ }^{3} 1982$, 227s.

37 Brummer 1964 (wie Anm. 34), 60. Chastel (wie Anm. 36), 229s. Fritz Saxl, Antike Götter in der Spätrenaissance. Ein Freskenzyklus und ein Discorso des Jacopo Zucchi, Leipzig / Berlin 1927, 23.

38 Saxl (wie Anm. 37), 23.

39 Vasari / Milanesi (wie Anm. 10), V, 195. Vgl. Medri 1992 (wie Anm. 13). Zu Lorenzos Bauprojekt: Philip Ellis Foster, A Study of Lorenzo de' Medici's Villa at Poggio a Caiano, 2 Bde., New York / London 1978. L'Architettura di Lorenzo il Magnifico, hg. von Gabriele Morolli u. a., Ausst.-Kat., Mailand 1992, 88-102.

40 Lorenzo de' Medici, Opere, hg. von Attilio Simioni, II, Bari 1914, 53. Saxl (wie Anm. 37), 23s. Panofsky (wie Anm. 33), 303. Chastel (wie Anm. 36), 229s. Brummer 1964 (wie Anm. 34), 59ss. Luba Freedman, Once more Signorelli's „Pan Deus Arcadiae“, in: Konsthistorisk Tidskrift, 54, 1985, 152159.

41 Chastel (wie Anm. 36), 228.

42. Panofsky (wie Anm. 33), 303s. Gustavo Costa, La leggenda dei secoli d'oro nella letteratura italiana, Bari 1972, 68s. Kidwell 1993 (wie Anm. 35), 9.

43 Zitiert bei Costa (wie Anm. 42), 50s. Ovid, Metamorphosen, I, 89ss.

44 Boccaccio (wie Anm. 18), 72-75.

45 Cox-Rẹarick (wie Anm. 2), 150.

46 Kliemann 1972 (wie Anm. 2), 304-309. Id. 1976 (wie Anm. 2), 51-55.

47 Kliemann 1972 (wie Anm. 2), 311s. Winner 1972 (wie Anm. 2), 166-168.

48 Ragionamento di Mons. Paolo Giovio sopra i motti \& disegni d'arme, \& d'amore, che communemente chiamano Imprese. Con un Discorso di Girolamo Ruscelli, intorno allo stesso soggetto, Venezia $1556,6$.

49 Giovio (wie Anm. 48), 6: „ch'ella non sia oscura, di sorte, c'habbia mestiero della Sibilla per interprete a volerla intendere; nè tanto chiara ch'ogni plebeo l'intenda“.

50 Die schriftliche Identifizierung der Figur wich der Signatur des Malers. Medri 1995 (wie Anm. 15, 15) 
löst „I. F. P.“ als „Iacobus Facebat Pontormus“ auf. Die darunterliegende Inschrift „PAN“ schimmert heute deutlich durch (Medri 1995, Abb. S. 21).

Siehe z. B. Wright (wie Anm. 32), 334-340.

Cox-Rearick (wie Anm. 2), 143-152.

Erkinger Schwarzenberg / Beatrice Paolozzi Strozzi, Norzia o la costante Fortuna - la lunetta di Alessandro Allori a Poggio a Caiano, in: Kunst des Cinquecento in der Toskana (Italienische Forschungen, hg. vom Kunsthistorischen Institut in Florenz, 3. Folge, XVII), München 1992, 197-206, hier: 198202. In einem jüngeren Aufsatz (wie Anm. 1, 155) führt Schwarzenberg Belege dafür an, daß bereits in Festdekorationen für Leo X. wie in Alloris Fresko die Idee greifbar wird, das Rad der Fortuna anzuhalten und so die ewige Medici-Herrschaft herbeizuführen. Es gilt jedoch zu bedenken, daß im ephemeren Medium der Festdekoration eindeutigere propagandistische Aussagen möglich waren als in einem Freskenzyklus; da sich die aus Leos Zeit in Poggio a Caiano erhaltenen Fresken durch vorsichtig verschlüsselte politische Stellungnahmen auszeichnen, ist es unwahrscheinlich, daß Giovio in der einen Lünette offen das ewige Medici-Prinzipat anpreisen wollte.

54 Das hätte wohl der damaligen Politik des Kardinals Giulio de' Medici widersprochen, der auf Besänftigung der Opposition zielte; vgl. Rudolf von Albertini, Das florentinische Staatsbewußtsein im Übergang von der Republik zum Prinzipat, Bern 1955, 40, 42. J. N. Stephens, The Fall of the Florentine Republic (1512-1530), Oxford 1983, 109-112. Leopold D. Ettlinger, Hercules Florentinus, in: Mitteilungen des Kunsthistorischen Institutes in Florenz, 26, 1972, 119-142. Die nach der Vertreibung der Medici 1495 erfolgte Überführung von Herkules-Darstellungen aus dem Palazzo Medici in den Palast der Stadtregierung zeigt, daß die Republik sich den usurpierten Herkules zurückholen wollte (Ettlinger, 119).

55 Schwarzenberg 1992 (wie Anm. 53), Anm. 28.

56 Während Uffizi $454 \mathrm{~F}$ eindeutig die VertumnusLünette vorbereitet, wurde Uffizi $455 \mathrm{~F}$ verschiedentlich als Entwurf für die zweite Lünette angesprochen, jedoch nicht überzeugend gedeutet. Falciani 1996 (wie Anm. 14, 113s) betont das zutiefst Rätselhafte der Szene. Galluzzi interpretiert die Skizze als Eröffnungszeremonie des traditionellen Florentiner Festes Calendimaggio „con la presenza di buffoni, musicanti e personaggi in costume generalmente ,all'antica', con intenti parodistici“, während Bracciante die Zeichnung als „un'immagine degli incontri della Sacra Accademia Fiorentina" sieht. (Francesco Galluzzi, „... veddi le bagattelle ...". Su Pontormo a Poggio a Caiano, in: Bullettino storico empolese, 8, anno XXIX, 1985,
279-282, hier: 280. Anna Maria Bracciante, Ottaviano de' Medici e gli artisti, Florenz 1984, 60s. Cox-Rearick 1981 (wie Anm. 25), Cat. 131 und 132, Abb. 123 und 124. Beide Deutungen ignorieren die Atmosphäre von Furcht und Bedrohung, die in Uffizi $455 \mathrm{~F}$ von den zwei stehenden Figuren ausgeht. Plausibler ist daher Kliemanns auf Ovid gestützter Erklärungsversuch, es handle sich um eine alternative Vorstudie zur Vertumnus-Lünette, die zeige, wie Pomona vor dem in Gestalt eines vulgären Ochsentreibers auftretenden Vertumnus fliehe (Kliemann 1976, wie Anm. 2, 71s). Es könnte auch der von Properz erwähnte weinlaubbekränzte, betrunkene Vertumnus gemeint sein (vgl. Winner 1972, wie Anm. 2, 169).

57 Vasari / Milanesi (wie Anm. 10), V, 196; VI, 275s, 277s. Wie bei verschiedenen anderen Ausmalungsprojekten der 1530er Jahre wollte Pontormo laut Vasari den jungen Bronzino als seinen Gehilfen heranziehen (vgl. Edi Baccheschi, L'opera completa del Bronzino, Mailand 1973, 83).

58 Vasari / Milanesi (wie Anm. 10), VI, 264.

59 Vasari / Milanesi (wie Anm. 10), V, 35; VI, 275.

60 Vasari / Milanesi (wie Anm. 10), VI, 276.

61 Zum „calcio“ siehe Horst Bredekamp, Florentiner Fußball. Die Renaissance der Spiele. Calcio als Fest der Medici, Frankfurt / New York 1993.

62 Luisa Marcucci, La „Maniera“ del Pontormo (Quaderni Pontormeschi 3), Florenz 1956, 20. Cox-Rearick lehnt diese Identifizierung aus stilistischen Gründen ab (Cox-Rearick 1981, wie Anm. 25, Cat. 343); sie erklärt den "Adonis“ als Vorzeichnung für die weiblichen Allegorien in Castello, was aber wegen der Zerstörung der CastelloFresken nicht zu verifizieren ist. Falciani 1996 (wie Anm. 14, 158s) referiert beide Vorschläge, ohne sich für einen davon zu entscheiden. Vasari (zitiert bei Cox-Rearick, wie Anm. 2, 260) charakterisiert Pontormos Figuren in Castello als ,molto difforme, e certi stravolgimenti ed attitudini che vi sono, pare che siano senza misura e molto strane" - dies trifft wohl kaum auf die besprochene „Adonis“Zeichnung zu.

63 Cox-Rearick 1981 (wie Anm. 25, Cat. 312 und 314, Abb. 302) erkennt in dieser Zeichnung eine „Prudentia“ für die zerstörte Loggia in Careggi. Im Gegensatz zu der Prudentia-Allegorie in der Casa Vasari, die Cox-Rearick zum Vergleich heranzieht, fehlt Pontormos „Prudentia“ allerdings die Schlange als Attribut. Der Spiegel allein reicht nicht für die Identifizierung als Prudentia aus, da er ja auch ein geläufiges Venus-Attribut ist (siehe z. B. Philostrat, Eikones, Buch 1, 6 und die venezianischen Venus- bzw. Kurtisanenbilder).

64 Falciani 1992 (wie Anm. 14), 120; wiederholt bei Falciani 1996 (wie Anm. 14), 101. 
65 Ovid, Metamorphoseos, Lyon 1518, Reprint New York / London 1976, fol. CXL verso. (Die Erstausgabe dieser bis in die 1580er Jahre hinein immer wieder neu aufgelegten kommentierten Edition erschien 1493 in Venedig.)

66 W. H. Roscher, Ausführliches Lexikon der griechischen und römischen Mythologie, Leipzig 18841886, I, Sp. 69-76. James George Frazer, Adonis Attis Osiris. Studies in the History of Oriental Religion, London 1955, I, 223-229.

67 Francesco Colonna, Hypnerotomachia Poliphili. Edizione critica e commento, hg. von Giovanni Pozzi / Lucia A. Ciapponi, Padua 1964, I, 363-371. Olimpia Pelosi, Il sogno di Polifilo - una quête dell'umanesimo, Salerno ${ }^{2}$ 1988, 47, 71, 77s, 134. Walter Friedlaender, La tintura delle rose, in: The Art Bulletin, 20, 1938, 320-324. Linda Fierz-David, Der Liebestraum des Poliphilo. Ein Beitrag zur Psychologie der Renaissance und der Moderne, Zürich 1947, 184, 197s. Edgar Wind, Pagan Mysteries in the Renaissance, London 1958, 104, 147. E. Kretzulesco-Quaranta, Les Jardins du Songe, 60, 217-219.

68 Cox-Rearick (wie Anm. 2), 178.

69 Wright (wie Anm. 32), 829-831. Giovanni Boccaccio, Genealogiae, Venedig 1494, Reprint New York / London 1976, Liber Secundus, Cap. LIII (20 verso). Ioannis Pierii Valeriani Bellunensis Hieroglyphica (...), Lyon 1602, 88.

70 In: Joannes Jovianus Pontanus, Urania u. a., Florenz 1514, 139ss (in Auszügen abgedruckt im Anhang dieses Beitrags). In italienischer Übersetzung als Giovanni Pontano, Gli orti delle Esperidi, Venezia 1761.

71 Carol Kidwell, Pontano. Poet and Prime Minister, London 1991, 130, 185, 190-192, 202, 385.

72 Kidwell 1991 (wie Anm. 71), 229, 233. J. R. Hale, Florence and the Medici. The Pattern of Control, Plymouth 1977, 77. Ausführlich, aber rein aus französischer Sicht: H.-François Delaborde, L'Expédition de Charles VIII en Italie. Histoire diplomatique et militaire, Paris 1888.

73 Erasmo Pèrcopo, Lettere di Giovanni Pontano a Principi ed Amici. Memoria letta all'Accademia Pontaniana nella tornata dell' 11 Novembre 1906, 75.

74 Giovanni Pontano, I trattati delle virtù sociali. De Liberalitate, De Beneficentia, De Magnificentia, De Splendore, De Conviventia, hg. von Francesco Tateo, Rom 1965, XVI, 196, 245-248.

75 Zu Piero siehe Paolo Giovio, Gli Elogi. Vite brevemente scritte d'huomini illustri di guerra, antichi et moderni (...) tradotte per M. Lodovico Domenichi, Florenz 1554, 215. E. Grassellini / A. Fracassini, Profili Medicei. Origine, sviluppo, decadenza della famiglia Medici attraverso i suoi componenti,
Florenz 1982, 225 (mit Bibliographie). Kidwell (wie Anm. 71), 233, 245. Zu Pieros Politik: Christopher Hibbert, The Rise and Fall of the House of Medici, London 1974, 177-188. Nicolai Rubinstein, The Government of Florence under the Medici (1434 to 1494), Oxford 1966, 229-235.

76 Zur Zitruspflanzenzucht der Medici: Schwarzenberg (wie Anm. 47), 197. Horst Bredekamp, Botticelli. Primavera. Florenz als Garten der Venus, Frankfurt a. M. 1988, 51.

77 Bredekamp (wie Anm. 76), 6.

78 Paolo Giovio, Le Iscrittioni poste sotto le vere Imagini de gli Huomini Famosi in Lettere. Tradotte di Latino in Volgare da Hippolito Orio Ferrarese, Venedig 1558, 101.

79 Giovio (wie Anm. 48), 6.

80 Kliemann 1976 (wie Anm. 2), 84. Vgl. Hans Martin von Erffa, Meditationen über die Palla Medicea, in: Festschrift Ulrich Middeldorf, hg. von Antje Kosegarten / Peter Tigler, Berlin 1968, 392-401, hier: 396.

81 Mirella Levi d'Ancona, Botticelli's Primavera. A botanical interpretation including astrology, alchemy and the Medici, Florenz 1983, 89.

82 Mirella Levi d'Ancona, The Garden of the Renaissance. Botanical Symbolism in Italian Painting, Florenz 1977, 205, 272s. Ead. 1983 (wie Anm. 81), 87ss. Plinius, Naturalis Historiae Libri XXXVII, hg. von Roderich König / Gerhard Winkler, Buch XII, Kempten 1977, Kap. VII; Buch XIII, Kempten 1977, Kap. XXIX-XXXI; Buch XXIII, München 1993, Kap. XLV, LVI.

83 Francis Ames-Lewis, Early Medicean Devices, in: Journal of the Warburg and Courtauld Institutes, 42, 1979, 122-143, hier: 128.

84 Bredekamp 1988 (wie Anm. 76), 51-55. AmesLewis (wie Anm. 83), 128.

85 Winner 1963/64 (wie Anm. 2), 10.

86 Hans Henrik Brummer, The Statue Court in the Vatican Belvedere, Stockholm 1970, 238. Kliemann 1976 (wie Anm. 2), 84. Cox-Rearick (wie Anm. 2), 151. Tobias Leuker, Numismatische Variationen über das Thema des "Goldenen Apfels“. Zu einer berühmten Medaille für Cosimo Pater Patriae, in: Florenz - Rom: Zwischen Kontinuität und Konkurrenz. Akten des am 10./11. April 1997 am Kunsthistorischen Institut in Florenz veranstalteten interdisziplinären Kolloquiums, hg. von Henry Keazor, Münster 1998, 71-84, hier: 76-78.

87 Brummer (wie Anm. 86), 233. Cox-Rearick (wie Anm. 2), 47s, 118, 144s, 150 s.

88 Robert von Ranke-Graves, Griechische Mythologie. Quellen und Deutung, II, Reinbek 1955, 138 s. Levi d'Ancona 1977 (wie Anm. 82), 207.

89 Vgl. Anm. 5.

90 Kliemann 1976 (wie Anm. 2), 84. 
91 Zum Motto „semper“: Ames-Lewis (wie Anm. 83), 135, 137. Cox-Rearick (wie Anm. 2), 16 ss. Franco Cardini, Le Insegne Laurenziane, in: Le Tems Revient. 'L Tempo si rinuova. Feste e spettacoli nella Firenze di Lorenzo il Magnifico, hg. von Paola Ventrone, Ausst.-Kat., Mailand 1992, 55-74.

92 Kidwell (wie Anm. 71), 225.

93 Pontano beschreibt, daß man einen Zitronenbaum überwintern kann, indem man ihn mit Wasser besprengt, so daß das sich bildende Eis die Wärme im Innern des Baumes einschließt. Für dieses Faktum wählt Pontano ein Bild, das biologisch unmöglich ist, aber genau auf zwei bekannte Medici-Impresen anspielt: Der Baum brennt im Innern („furit intus flamma vorax“, „siccas occulto ex igne medullas“). Sowohl Giuliano als auch Piero de’ Medici führten als corpus ihrer Imprese einen Broncone, der innerlich brennt, so daß an den Schnittstellen Rauch aus dem Zweig austritt. Pontano paraphrasiert quasi die zugehörigen Motti, nämlich „par le fue (feu) reverdira“ bzw. „in viridi teneras exurit flamma medullas“. Zu den Impresen: Paolo Giovio, Dialogo dell'imprese militari e amorose, hg. von Maria Luisa Doglio, Rom 1978, 64. Salvatore Settis, Citarea „su una impresa di bronconi“, in: Journal of the Warburg and Courtauld Institutes, 34, 1971, 135-177, hier: 136. Cox-Rearick (wie Anm. 2), 22s.

94 Daß das Gedicht erstmals 1505 mit einer Widmung an Francesco Gonzaga erschien (Kidwell, wie Anm. 71, 294, 404), spricht nicht notwendigerweise gegen meine Hypothese, denn zur Zeit des Medici-Exils war eine Widmung an die Medici wirklich nicht mehr opportun. Die kurze Widmungsstrophe für Gonzaga ist ein isoliertes Element im Text, das leicht gegen eine frühere andere Widmung ausgetauscht worden sein könnte. In den Impresen und Wappen von Francesco Gonzaga spielen weder Lorbeer noch Hesperidenbäume eine Rolle. Vgl. Giancarlo Malacarne, Araldica Gonzaghesca. La storia attraverso i simboli, Ausst.-Kat., Modena 1992, 105ss.

95 Ute Davitt Asmus, Corpus Quasi Vas. Beiträge zur Ikonographie der italienischen Renaissance, Berlin $1977,90$.

96 Giovio (wie Anm. 78), 101s. Reduziertere lateinische Version in Paolo Giovio, Gli elogi degli uomini illustri (letterati - artisti - uomini d'arme), hg. von Renzo Meregazzi (Pauli Iovii Opera, VIII), Rom 1972, 76s.

97 Siehe oben Anm. 78.

98 So sind z. B. für Valeriano Zitronen Hieroglyphen der „aeternitas“ (Valeriano, wie Anm. 69, 541, 644). Lorbeer als „Symbol der Beständigkeit“ (Kliemann 1976, wie Anm. 2, 48).

99 Aby Warburg, Sandro Botticellis „Geburt der Venus“ und „Frühling“. Eine Untersuchung über die
Vorstellungen von der Antike in der italienischen Frührenaissance, Hamburg / Leipzig 1893, 3s, 11. Charles Dempsey, Mercurius Ver: The Sources of Botticelli's Primavera, in: Journal of the Warburg and Courtauld Institutes, 31, 1968, 251-273, hier: 257, 260, Anm. 42, 264s.

100 Ovid, Fasti, IV, 1-18, 85-132. Dempsey (wie Anm. 99), 257s, 261ss, 267. Cox-Rearick (wie Anm. 2), 131.

101 Dempsey (wie Anm. 99), 257, 258, 261-264, 267. Wright (wie Anm. 32), 341-346. Bredekamp (wie Anm. 76). Michael Rohlmann, Botticellis „Primavera“. Zu Anlaß, Adressat und Funktion von mythologischen Gemälden im Florentiner Quattrocento, in: Artibus et historiae, 33, 1996, 97-132.

102 Medri (wie Anm. 15), 15.

103 Das Motto für die Hesperiden-Lünette hätte man z. B. aus dem Umkreis von Vergils Passus über Zitronenbäume entnehmen können (Georgica, II, 126ss).

104 Harry Levin, The myth of the Golden Age in the Renaissance, Bloomington / London 1969, 194.

105 Ovid, Metamorphosen, I, 107. Angelus Politianus, Opera Omnia, hg. von Ida Maier, III: Opera Miscellanea et Epistulae, Turin 1971, 28s. Levin (wie Anm. 104), 21. Costa (wie Anm. 42), 53, 65, 69.

106 Wind (wie Anm. 67), 84. Brummer (wie Anm. 86), 230-233. Cox-Rearick (wie Anm. 2), 150.

107 Politianus (wie Anm. 105), 36.

108 Cox-Rearick (wie Anm. 2), 144, 150.

109 Bredekamp (wie Anm. 76), 53, 84.

110 Schwarzenberg (wie Anm. 1), 147.

111 Laut Wright (wie Anm. 32, 349) bildete das von Vasari überlieferte Projekt für Poggio a Caiano die Programmgrundlage zum Venusgarten der Medici-Villa Olmo a Castello.

112 Die Maskerade „Genealogia degli Dei“ fand 1566 im Kontext der Hochzeitsfeiern für Francesco de’ Medici statt. Die gründlichste Untersuchung dieser Feiern, die von Borghini entscheidend mitgestaltet wurden, findet sich bei Randolph Starn / Loren Partridge, Arts of Power. Three Halls of State in Italy, 1300-1600, Berkeley / Oxford 1992, 151-212, 267-294. Da Borghini damals mit allen wichtigen Medici-Projekten zu tun hatte, sieht Seznec ihn ohne weiteres auch als den Organisator der Maskerade. (Jean Seznec, Das Fortleben der antiken Götter. Die mythologische Tradition im Humanismus und in der Kunst der Renaissance, München 1990, 216s.) Scorza weist allerdings darauf hin, daß es dafür keine Beweise gibt. (R. A. Scorza, Vincenzo Borghini and „Invenzione“: The Florentine „Apparato“ of 1565, in: Journal of the Warburg and Courtauld Institutes, 44, 1981, 57-75, hier: 58.) 
113 Baccio Baldini, Discorso sopra la Mascherata della Genealogia degl'Iddei de' Gentili, Florenz 1565, 51. Feste e apparati medicei da Cosimo I a Cosimo II, hg. von Giovanna Gaeta Bertelà / Annamaria Petrioli Tofani, Ausst.-Kat., Florenz 1969, 19, 2123.

114 Borghini „amicissimo di Jacopo“ (Pontormo): Vasari / Milanesi (wie Anm. 10), VI, 275. Allgemein zum Verhältnis Vasari / Borghini: Robert Joseph Williams, Vincenzo Borghini and Vasari’s „Lives“, Diss. Princeton 1988. Zu Borghinis Kenntnis von Giovios Programm: Susan Regan McKillop, Franciabigio, Berkeley 1974, 73. Kliemann 1976 (wie Anm. 2), 88. Cox-Rearick (wie Anm. 2), 105ss.

115 Zur Allori-Lünette: Schwarzenberg / Strozzi (wie Anm. 53).

116 Vasari / Milanesi (wie Anm. 10), V, 195.

117 Bardazzi / Castellani (wie Anm. 31), 445, Abb. 435.

118 Winner 1970 (wie Anm. 2), 274s, 283-287. Kliemann 1976 (wie Anm. 2), 15-24. Kliemann 1985 (wie Anm. 2), 199s.

119 Brummer (wie Anm. 86), 232-238.

120 Kliemann 1976 (wie Anm. 2), 53, 84, Anm. 205. Cox-Rearick (wie Anm. 2), 118s, 151.

121 Bei Papst Leos Tod sei eine mächtige Zitronenhekke vor der römischen Villa Medici in sich zusammengesunken, trotz des strengen Winters aber nicht (wie alle anderen Bäume dieser Art) abgestorben, sondern bei der Wahl Giulio de' Medicis zum Papst wieder aufgelebt und neu emporge-

\section{Anhang}

Aus Giovanni Pontano: De Hortis Hesperidum (Joannes Jovianus Pontanus: Urania u. a., Florentiae $1514,139 \mathrm{ff}$.)

Qualis sit arbor citrius, E unde oriunda. Orbe etenim Hesperio. ni asiq; ad littora quondam Oceani, auriferis primum sese extulit hortis Citrius, arboreae referens preaconia palmae, Illi perpetuus frondis decor. inter opacum Albescunt nitidi flores nemus. atq; ita late Spirat odoratus Zephyris felicibus aer. Ipsa quidem Lauro foliisq; \& cortice, \& ipso Stipite tum similis, tum frondescente iuventa, At cono inferior, ramisq; valentibus impar, Nam florum longe candore, \& odoribus anteit. Quin gravida e ramis, triplici \& distincta colore Mala nitent, virides primum referentia frondes, Hinc rutilant, fulvoq; micant matura metallo, wachsen, behauptete Valeriano. Eine merkwürdig enge, wahrscheinlich panegyrisch konstruierte Parallele zu der von Pontano beschriebenen wundersamen Regeneration des hesperidischen Zitronenbaumes! Valeriano zitiert bei Christel Meier / Nikolaus Staubach, David Orans. Ein unbekanntes Goldemail des Medici-Papstes Leo X., in: Iconologia Sacra. Mythos, Bildkunst und Dichtung in der Religions- und Sozialgeschichte Alteuropas. Festschrift für Karl Hauck zum 75. Geburtstag, hg. von Hagen Keller / Nikolaus Staubach, Berlin / New York 1994, 569-624, hier: 622.

122 T. C. Price Zimmermann, Paolo Giovio. The Historian and the Crisis of Sixteenth-Century Italy, Princeton 1995, 15.

123 Textquellen zum leoninischen Goldenen Zeitalter bei Kliemann 1976 (wie Anm. 2), 241ss. Siehe auch Ernst Hans Gombrich, Renaissance and Golden Age, in: Journal of the Warburg and Courtauld Institutes, 24, 1961, 306-309.

124 Zur Leo-Selbstdarstellung als Friedensbringer und "christus medicus“ siehe Rudolf Preimesberger, Tragische Motive in Raffaels „Transfiguration“, in: Zeitschrift für Kunstgeschichte, 50, 1987, 89-115, hier: 95. Claudia Rousseau, The Yoke Impresa of Leo X, in: Mitteilungen des Kunsthistorischen Institutes in Florenz, 33, 1989, 113-126, hier: 116s, $122 \mathrm{~s}$.

125 Ames-Lewis (wie Anm. 83), 141. Kliemann 1972 (wie Anm. 2), 308.

Flore novo semper, semper quoq; foetibus aucta, Perpetuum Veneris monumentum, at triste dolorum.

De conversione Adonidis in citrium.

Moerebat puero extincto, lugebat, amantem

Scissa comam, \& lacrimis humebat terra profusis.

Humebant Lauri, quarum frondente sub umbra.

Et positum ante pedes lamentabatur Adonim,

Et se oblita deam, tundebat pectora palmis.

Ut vero ses dolor, \& gravis ira repressit,

Ac veterum admonuit Daphne Peneia amorum,

Et nostros inquit testabitur arbor amores,

Nostrorum \& maneant monimenta aeterna doloru.

Ambrosio mox rore comam diffundit, \& unda

Idalia corpus lavit, incomperataq; \& oscula iungit.

Ambrosium sensit rorem coma, sensit \& undam

Idaliam corpus, divinaq; verba loquentis.

Haeserunt terrae crines, riguitq; capillus 
Protenta in radice, \& recto in stipite corpus, Languo in teneras abiit mollissima frondes, In florem candor, in ramos brachia, \& ille, Ille decor tota diffusus in arbore risit.

Vulnificos spinae, referunt in cortice dentes. Crescit $\&$ in patulas Aphrodisia Citrius umbras.

Colligit hinc sparsos crines Dea, mandat \& altae

Telluri infodiens, tum sic affata: Meis heu Consita de lacrimis, nunq viduabere fronde, Semper flore novo, semperq; ornabere pomis Hortorum decus, \& nemorum, illecebraeq; domorum, Osculaq; illacrimans ligno dedit, eq; capillis Summa sub tellure agitans, fibramina ducit, Hauriat ut sitiens undam, atq; alimenta ministret. Illa velut dominae luctum solata, recenteis Excussit frondes, resupinaq; vertice, canos Diffudit florum nimbos, queis pectora divae Implevitq; sinum, \& lacrimas sedavit eunteis. Ex in Hesperiis arbor nitet aurea sylvis. $[\ldots]$

\section{Abbildungsnachweis}

\section{Alinari: 1, 6}

Archiv der Autorin: 2, 3
Fabula de Citriorum reparatione, novaq; apportatione in Italiam, e media regione.

Nanq; ferunt ob primitias quandoq; negatas Iunonem indignantem animo, graviterq; dolentem Immisse \& stragem arboribus, citriisq; ruinam, Hesperii generis malorum stirpe perempta. Donec eam Venus e Medorum divite sylva Relliquias relegens, per Aphrorum pinguia culta Rursus in Italiam genti trasmisit habendam Aeneadum, quo priscus honos, \& cura nepotum in sylvis veteres renovaret Adonidis ignes, Aeternum \& maneant monumenta insignia amoru. Tu vero quoties aestusq; \& solis iniquum Fervorem metues, rapidi \& fera sydera cancri, Aut glaciem quoties, inimicae $\&$ tempora brumae, Verge iterum latices, iterum fluvialia mersa Stagna super. sic ipse aestus, sic frigora vinces. Nanq; aestusitientem alte, externoq; calore Arentem madidans, sedas aestumq; sitimq; At glacie inclusus calor aestuat, \& furit intus Flama vorax. tum funde imbre, tum flumine largo Irrora ficcas occulto ex igne medullas, Quo vigor adversum soles se \& frigora duret.

Florenz, Soprintendenza per i beni artistici e storici: 4, 5, 7 Hamburger Kunsthalle: 8 